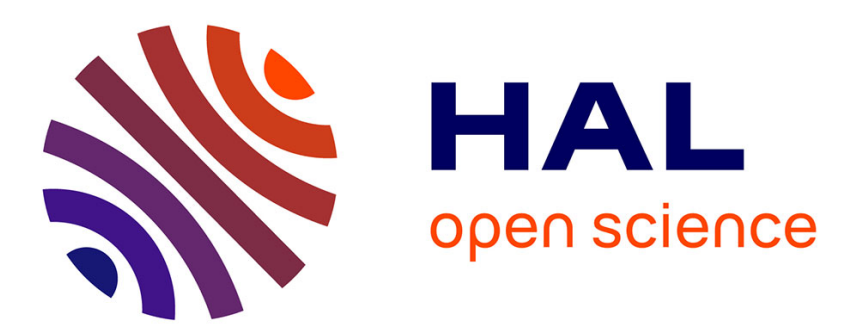

\title{
Casimir-Polder force and torque for anisotropic molecules close to conducting planes and their effects on CO_2
}

\author{
Mauro Antezza, Ignat Fialkovsky, Nail Khusnutdinov
}

\section{- To cite this version:}

Mauro Antezza, Ignat Fialkovsky, Nail Khusnutdinov. Casimir-Polder force and torque for anisotropic molecules close to conducting planes and their effects on CO_2. Phys.Rev.B, 2020, 102 (19), pp.195422. 10.1103/PhysRevB.102.195422 . hal-02973233

\section{HAL Id: hal-02973233 \\ https://hal.science/hal-02973233}

Submitted on 25 May 2021

HAL is a multi-disciplinary open access archive for the deposit and dissemination of scientific research documents, whether they are published or not. The documents may come from teaching and research institutions in France or abroad, or from public or private research centers.
L'archive ouverte pluridisciplinaire HAL, est destinée au dépôt et à la diffusion de documents scientifiques de niveau recherche, publiés ou non, émanant des établissements d'enseignement et de recherche français ou étrangers, des laboratoires publics ou privés. 


\title{
Casimir-Polder force and torque for anisotropic molecules close to conducting planes and their effect on $\mathrm{CO}_{2}$
}

\author{
Mauro Antezza $\odot,{ }^{1,2, *}$ Ignat Fialkovsky $\odot,,^{3, \dagger}$ and Nail Khusnutdinov $\odot^{3,4, \hbar}$ \\ ${ }^{1}$ Université de Montpellier, Laboratoire Charles Coulomb, Place Eugène Bataillon CC074, F-34095 Montpellier Cedex 05, France \\ ${ }^{2}$ Institut Universitaire de France, 1 rue Descartes, F-75231 Paris, France \\ ${ }^{3}$ CMCC, Universidade Federal do ABC, Avenida dos Estados 5001, CEP 09210-580, São Paulo, Brazil \\ ${ }^{4}$ Regional Scientific and Educational Mathematical Center of Kazan Federal University, Kremlevskaya 18, Kazan 420008, Russia
}

(Received 5 October 2020; revised 29 October 2020; accepted 3 November 2020; published 17 November 2020)

\begin{abstract}
We derive the Casimir-Polder force and Casimir torque expressions for an anisotropic molecule close to a conducting plane with a tensorial conductivity. We apply our general expressions to the case of a carbon dioxide $\mathrm{CO}_{2}$ molecule close to a plane with pure Hall conductivity and to graphene. We show that the equilibrium position of this linear molecule is with its main axis perpendicular to the surface. We hence conjecture a possible way to exploit the Casimir torque to mechanically improve the performance of $\mathrm{CO}_{2}$ separation membranes useful for an efficient atmospheric $\mathrm{CO}_{2}$ reduction.
\end{abstract}

DOI: 10.1103/PhysRevB.102.195422

\section{INTRODUCTION}

The van der Waals-Casimir dispersion forces play an important role in different phenomena in physics and biology, as well as in chemistry [1-4]. The original consideration by Casimir [5], devoted to a force between two perfect metallic plates, has been developed to include different geometries of boundaries [1,6,7], nonequilibrium thermal conditions [8-12], and different materials [4] such as graphene [13,14], topological insulators [15,16], chiral metamaterials [17], and Weyl semimetals [18].

Casimir and Polder also derived [19] the force between an atom in the ground state and a perfectly conducting plate, taking into account the retardation of electromagnetic interactions. In a nonrelativistic case, this force is the van der Waals one based on the London interaction between atoms. There exist plenty of different approaches for calculation of van der Waals-Casimir and Casimir-Polder energies and forces both at zero and nonzero temperature (see Refs. $[1-4,12,20]$ for details and further references). Lifshitz [21] was among the first who suggested a method of calculation of the Casimir-Polder force based on the consideration of vacuum fluctuations of the electromagnetic field, as well as a rarefying procedure. This approach takes into account a simple observation that for a rarefied media the dielectric permittivity $\varepsilon \approx 1+4 \pi N \alpha$, where $N$ is the number of atoms in a unit volume and $\alpha$ is the polarizability of a single atom.

The van der Waals-Casimir and Casimir-Polder effects for anisotropic molecules have been the subject of intensive investigation in the recent years [22-24]. The molecule

\footnotetext{
*mauro.antezza@umontpellier.fr

†ifialk@gmail.com

‡nail.khusnutdinov@gmail.com
}

anisotropy gives life to a new phenomenon: the Casimir torque effect, which was first predicted in Refs. [25] (see also [26] and references therein). The Casimir energy for isotropic molecules naturally does not depend on the orientation of the latter in space, which is not the case for anisotropic molecules. The momentum of force appears which is called the Casimir torque. A molecule then is being rotated to assume a specific position in space in relation to the boundary with minimum energy and zero torque. The carbon dioxide molecule $\mathrm{CO}_{2}$, for example [23], has an equilibrium position perpendicular to the dielectric slab, i.e., with the direction of the larger polarizability being perpendicular to the surface.

The polarizability tensor of a molecule or atom has, in general, both symmetric and antisymmetric contributions [27]. The antisymmetric part is accounted for by atomic hyperfine structure $[28,29]$ and is defined by total angular momentum of the molecule or atom. This part of polarizability gives a contribution to the $P$-odd van der Waals forces [29] (see also review [30]) and when interacting with a Chern-Simons surface [24].

By using the rarefying Lifshitz procedure together with the scattering matrix approach, we achieve two goals in this paper: (i) we obtain a general expression for Casimir-Polder force for anisotropic molecules near the conductive plane with arbitrary tensorial conductivity, and (ii) we obtain a general expression for Casimir torque in this configuration. Moreover, we apply these general expressions for molecule $\mathrm{CO}_{2}$ near a plane with pure Hall conductivity and in front of freestanding graphene. The focus on studying the carbon dioxide molecules interacting with graphene originates from growing research in possible ways of implementation of graphene membranes for gas separation [31] (and water desalination [32]) aimed to diminish the $\mathrm{CO}_{2}$ footprint of industry and to separate the gas from the atmosphere. Our predictions show that the Casimir torque tends to orient the $\mathrm{CO}_{2}$ molecules in a position favoring 


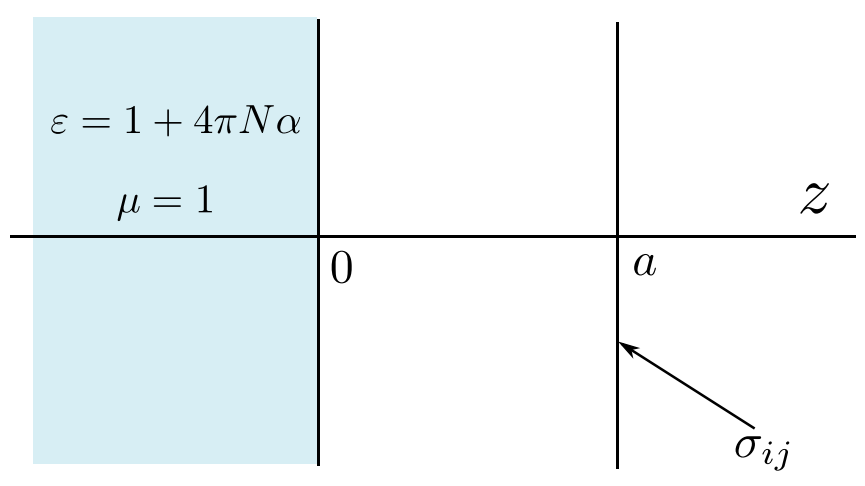

FIG. 1. A conductive plane with conductivity tensor $\sigma$ is situated at $z=a$. Semispace $z \leqslant 0$ is filled by a nonmagnetic dielectric. We calculate Casimir energy for this system and then rarefy the dielectric, that is we set $\varepsilon=1+4 \pi N \alpha$ and put $N \rightarrow 0$. The CP energy is given by Eq. (1) for plane and atom at $z=0$.

their passage through the membrane, facing the latter by the molecules' smallest facet. Thus, possible enhancements of the effect may lead to the improvement of the efficiency of graphene-based membranes in gas separation applications.

The paper is organized in the following manner. In Sec. II we consider an isotropic atom near a conductive plane and implicate different forms of tensorial conductivity. Section III is devoted to the derivation of the Casimir-Polder energy for anisotropic atoms or molecules in front of a conductive plane with arbitrary tensorial conductivity. We extract in a manifest form the contributions due to symmetric and antisymmetric parts of polarizability and consider some specific cases. In Sec. IV we derive the general form of Casimir torque and apply it for the $\mathrm{CO}_{2}$ molecule near a surface with pure Hall conductivity and in front of graphene. We finish in Sec. V with a discussion and summarizing our results. Some technical details of the calculations are given in the Appendix.

Throughout of the paper we use the natural units $\hbar=c=$ 1 ; the greek indices $\mu, v=1,2,3=x, y, z$ and the latin ones $i, j=1,2=x, y$.

\section{ISOTROPIC ATOMS}

To calculate Casimir-Polder (CP) energy we use the rarefying procedure of Lifshitz [21]. Towards this end let us consider a conductive plane positioned perpendicular to the axis $z$ at point $z=a$ and semi-infinite nonmagnetic dielectric media filling semispace $z \leqslant 0$ with dielectric permittivity $\varepsilon$ (see Fig. 1). Then we rarefy the dielectric by considering $\varepsilon=1+4 \pi N \alpha$ with $N \rightarrow 0$. The CP energy is given by the relation

$$
\mathcal{E}_{\mathrm{CP}}=-\lim _{N \rightarrow 0} \frac{1}{N} \frac{\partial \mathcal{E}_{\mathrm{C}}}{\partial a},
$$

where $\mathcal{E}_{\mathrm{C}}$ is the Casimir energy calculated for the system "conductive plane-dielectric."

To calculate Casimir energy $\mathcal{E}_{\mathrm{C}}$ for this system we use the expression obtained in Ref. [33] (see also Ref. [34]) and generalized in Ref. [35]:

$$
\mathcal{E}_{\mathrm{C}}=\iint \frac{d^{2} k}{2(2 \pi)^{3}} \int_{-\infty}^{\infty} d \xi \ln \operatorname{det}\left[\mathbf{I}-e^{-2 a \kappa} \mathbf{r}_{0}^{\prime}(i s \kappa) \mathbf{r}_{a}(i s \kappa)\right],
$$

where $\kappa=\sqrt{\xi^{2}+\boldsymbol{k}^{2}}, \omega=i \xi$, and $s=\operatorname{sgn} \xi$. Here $\mathbf{r}_{a}$ and $\mathbf{r}_{0}^{\prime}$ are $2 \times 2$ reflection matrices of the plane and the dielectric.

The boundary conditions give the following expressions for reflection matrices (we denote two-dimensional tensors in bold):

$$
\begin{aligned}
& \mathbf{r}_{0}^{\prime}(i s \kappa)=\frac{1-\varepsilon}{\varepsilon+1+\varepsilon \frac{\kappa}{\kappa_{\varepsilon}}+\frac{\kappa_{\varepsilon}}{\kappa}}\left\{\left(1-\frac{\boldsymbol{k}^{2}}{\kappa_{\varepsilon}^{2}}\right) \mathbf{I}+2 \frac{\boldsymbol{k} \otimes \boldsymbol{k}}{\kappa_{\varepsilon}^{2}}\right\}, \\
& \mathbf{r}_{a}(i s \kappa)=-\frac{\xi^{2} \boldsymbol{\eta}+\boldsymbol{k} \otimes(\boldsymbol{k} \boldsymbol{\eta})+\mathbf{I} \kappa|\xi| \operatorname{det} \boldsymbol{\eta}}{\xi^{2} \operatorname{tr} \boldsymbol{\eta}+\boldsymbol{k} \boldsymbol{k} \boldsymbol{\eta}+\kappa|\xi|(1+\operatorname{det} \boldsymbol{\eta})}
\end{aligned}
$$

where $\boldsymbol{\eta}=2 \pi \boldsymbol{\sigma},[\boldsymbol{k} \otimes(\boldsymbol{k} \boldsymbol{\eta})]_{j}^{i}=k^{i} k^{n} \eta_{n j}, \boldsymbol{k} \boldsymbol{k} \boldsymbol{\eta}=k^{i} k^{j} \eta_{i j}, \kappa_{\varepsilon}=$ $\sqrt{\varepsilon \xi^{2}+\boldsymbol{k}^{2}}$.

In the rarefying limit, $N \rightarrow 0$,

$$
\mathbf{r}_{0}^{\prime}(i s \kappa)=-\frac{\pi N \alpha}{\kappa^{2}}\left(\xi^{2} \mathbf{I}+2 \boldsymbol{k} \otimes \boldsymbol{k}\right)+O\left(N^{2}\right) .
$$

Taking into account the simple formula

$$
\operatorname{det}(1+\epsilon A)=1+\epsilon \operatorname{tr} A+O\left(\epsilon^{2}\right),
$$

we obtain the $\mathrm{CP}$ energy

$$
\mathcal{E}_{\mathrm{CP}}=\iint \frac{d^{2} k}{(2 \pi)^{2}} \int_{0}^{\infty} \frac{d \xi}{\kappa} \alpha(i \xi) e^{-2 a \kappa}\left(\xi^{2} \operatorname{tr} \mathbf{r}_{a}+2 \boldsymbol{k} \boldsymbol{k} \mathbf{r}_{a}\right),
$$

or, in the manifest form,

$$
\begin{aligned}
\mathcal{E}_{\mathrm{CP}}= & -\iint \frac{d^{2} k}{(2 \pi)^{2}} \int_{0}^{\infty} \frac{d \xi}{\kappa} \alpha(i \xi) e^{-2 a \kappa} \\
& \times \frac{\xi^{4} \operatorname{tr} \boldsymbol{\eta}+\boldsymbol{k} \boldsymbol{k} \boldsymbol{\eta}\left(\xi^{2}+2 \kappa^{2}\right)+2 \kappa^{3} \xi \operatorname{det} \boldsymbol{\eta}}{\xi^{2} \operatorname{tr} \boldsymbol{\eta}+\boldsymbol{k} \boldsymbol{k} \boldsymbol{\eta}+\kappa \xi(1+\operatorname{det} \boldsymbol{\eta})} .
\end{aligned}
$$

This expression is valid for an arbitrary form of the conductivity. For a plane with ideal conductivity, $\boldsymbol{\eta} \rightarrow \infty$, the $\mathrm{CP}$ energy tends to that for the ideal plane, $\mathcal{E}_{\mathrm{CP}} \rightarrow \mathcal{E}_{\mathrm{CP}}^{i d}$, where

$$
\mathcal{E}_{\mathrm{CP}}^{i d}=-\frac{1}{16 \pi a^{4}} \int_{0}^{\infty} d z e^{-z} \alpha\left(\frac{i z}{2 a}\right)\left(2+2 z+z^{2}\right) .
$$

For large distances between the atom and the plane we change integrand variables $\boldsymbol{k} \rightarrow \boldsymbol{k} / a, \xi \rightarrow \xi / a$ and take the limit $a \rightarrow \infty$. We observe that the CP energy is proportional to that for a perfect metal, $\mathcal{E}_{\mathrm{CP}}^{\infty}=-3 \alpha(0) / 8 \pi a^{4}$, with coefficient depending on the conductivity of the plane. This was observed in Ref. [36] for isotropic conductivity. Note that the actual parameter of expansion depends on the structure of the polarization tensor $\alpha$, which must include dimensionful parameters describing the properties of the atom or molecule; see (18).

Let us consider the general form of the conductivity tensor,

$$
\boldsymbol{\eta}=X \mathbf{I}+Y \frac{\boldsymbol{k} \otimes \boldsymbol{k}}{\boldsymbol{k}^{2}}+Z \boldsymbol{\epsilon},
$$

where all coefficients are functions of $(\xi, k)$ and $\epsilon$ is the totally antisymmetric tensor. In particular, the graphene conductivity tensor has such structure [13] with $X=\eta_{\mathrm{te}}, Y=\eta_{\mathrm{tm}}-\eta_{\mathrm{te}}$, 
and $Z=0$ [37]. Here $\eta_{\mathrm{tm}, \text { te }}$ are the conductivities of TM and TE modes, respectively. If $Z \neq 0$, the parity anomaly is present [35]. The pure Hall conductivity corresponds to the case with $X=Y=0$ and $Z \neq 0$.

Taking the polar coordinates for $\left(k_{1}, k_{2}\right)$ and integrating over angular variables we obtain that

$$
\begin{aligned}
\mathcal{E}_{\mathrm{CP}}= & -\iint_{0}^{\infty} \frac{k d k d \xi}{2 \pi \kappa} \frac{\alpha(i \xi)}{W} e^{-2 a \kappa}\left\{\kappa^{2}\left(2 \kappa^{2}-\xi^{2}\right)(X+Y)\right. \\
& \left.+2 \kappa^{3} \xi\left(X^{2}+X Y+Z^{2}\right)+\xi^{4} X\right\}
\end{aligned}
$$

where

$$
W=\kappa^{2}(X+Y)+\kappa \xi\left(X^{2}+X Y+Z^{2}+1\right)+X \xi^{2} .
$$

For isotropic conductivity $\boldsymbol{\eta}=X \mathbf{I}$ the CP energy,

$$
\begin{aligned}
\mathcal{E}_{\mathrm{CP}}= & -\int_{0}^{\infty} \frac{d z z^{3}}{2 \pi} \int_{0}^{1} d x \alpha(i x z) e^{-2 z a} \\
& \times\left\{\frac{X x^{3}}{X x+1}+\frac{X\left(2-x^{2}\right)}{X+x}\right\},
\end{aligned}
$$

coincides with expression obtained in Ref. [36]. Here $X=$ $X(x z)$.

Let us consider pure Hall conductivity $X=Y=0$ and $Z \neq$ 0 . For constant $Z$ one has

$$
\mathcal{E}_{\mathrm{CP}}=\frac{Z^{2}}{1+Z^{2}} \mathcal{E}_{\mathrm{CP}}^{i d}
$$

where the CP energy for the plane with ideal conductivity, $\mathcal{E}_{\mathrm{CP}}^{i d}$, is given by Eq. (8). The same result was obtained in Refs. [24] for CP interaction of an atom with a Chern-Simons plane. The parameter of Chern-Simons interaction in those references coincides exactly with the Hall conductivity $Z$ in the above formulas.

For a two-dimensional (2D) electron systems in a strong magnetic field $B$ we have $\sigma_{x x} \ll \sigma_{x y}$ (see Ref. [34]) and

$$
Z=2 \pi \sigma_{x y}=v \alpha_{\mathrm{QED}}
$$

where $v=2 \pi n /(e B)$ is the Landau-level filling factor and $\alpha_{\mathrm{QED}}$ is the fine structure constant. The leading contribution over $\alpha_{\mathrm{QED}} \ll 1$ reads

$$
\mathcal{E}_{\mathrm{CP}}=Z^{2} \mathcal{E}_{\mathrm{CP}}^{i d}=\alpha_{\mathrm{QED}}^{2} v^{2} \mathcal{E}_{\mathrm{CP}}^{i d} .
$$

Therefore, the CP energy is quantized, too, alongside with Casimir energy [34] and suppressed by the same factor $\alpha_{\mathrm{QED}}^{2}$. The $\mathrm{CP}$ force is always attractive, for any distance and any value of the Hall conductivity. The detailed calculations for graphene in a strong magnetic field have already been done in Ref. [38].

In the general case with a combination of the isotropic and the Hall conductivities we set $Y=0$ and have

$$
\eta=X \mathbf{I}+Z \boldsymbol{\epsilon} .
$$

The CP energy reads

$$
\begin{aligned}
\frac{\mathcal{E}_{\mathrm{CP}}}{\mathcal{E}_{\mathrm{CP}}^{\infty}}= & \frac{4}{3} \int_{0}^{\infty} d y y^{3} \int_{0}^{1} d x \frac{\alpha(i x y / a)}{\alpha(0)} e^{-2 y} \\
& \times \frac{\left(2-x^{2}+x^{4}\right) X+2 x\left(X^{2}+Z^{2}\right)}{\left(1+x^{2}\right) X+x\left(1+X^{2}+Z^{2}\right)},
\end{aligned}
$$

where $\mathcal{E}_{\mathrm{CP}}^{\infty}=-3 \alpha(0) / 8 \pi a^{4}$ is the asymptotic of the CasimirPolder energy for the ideal plane (8) and the arguments of $X$ and $Y$ are replaced as $(\xi, k) \rightarrow\left(y x / a, y \sqrt{x^{2}-1} / a\right)$. The function $2-x^{2}+x^{4}$ is always positive in the domain of integration and therefore the CP energy is always negative and the $\mathrm{CP}$ force is attractive for any relation between $X$ and $Z$.

\section{ANISOTROPIC CASE}

To include in the consideration an anisotropic dynamic polarizability we consider a matter in semispace with tensorial dielectric permittivity $\varepsilon_{\mu \nu}$. First of all we have to consider the scattering problem for an anisotropic semispace $z \leqslant 0$ and vacuum for $z>0$ and then use the rarefying Lifshitz procedure: $\varepsilon_{\mu \nu}=\delta_{\mu \nu}+4 \pi N \alpha_{\mu \nu}$ with $N \rightarrow 0$.

In general, the polarizability tensor $\alpha_{\mu \nu}(\omega)$ may be expressed in terms of dipole matrix elements [27],

$$
\alpha_{\mu \nu}=\sum_{n}\left(\frac{D_{\mu \nu}^{n}}{\omega_{n 0}-\omega-i 0}+\frac{D_{\nu \mu}^{n}}{\omega_{n 0}+\omega+i 0}\right),
$$

where $\omega_{n 0}=\omega_{n}-\omega_{0}$ and $D_{\mu \nu}^{n}=\left\langle 0\left|\hat{d}_{\mu}\right| n\right\rangle\left\langle n\left|\hat{d}_{\nu}\right| 0\right\rangle$. The scattering tensor has the same form but with $-i 0$ in the second term [27]. For the case of elastic scattering [27], $\omega \neq \omega_{n 0}$ it is possible to omit $i 0$ in the denominator and the polarizability tensor becomes Hermitian. Note, that such an approximation, strictly speaking, violates the Kramers-Kronig relations. The polarizability tensor may be divided into symmetric and antisymmetric parts $\alpha_{\mu \nu}=\alpha_{\mu \nu}^{s}+\alpha_{\mu \nu}^{a}$, and for the elastic scattering case we have [24]

$$
\begin{aligned}
& \alpha_{\mu \nu}^{s}=\frac{\alpha_{\mu \nu}+\alpha_{\nu \mu}}{2}=\operatorname{Re} \alpha_{\mu \nu}=\sum_{n} \frac{2 \omega_{n 0} \operatorname{Re} D_{\mu \nu}^{n}}{\omega_{n 0}^{2}-\omega^{2}}, \\
& \alpha_{\mu \nu}^{a}=\frac{\alpha_{\mu \nu}-\alpha_{\nu \mu}}{2}=i \operatorname{Im} \alpha_{\mu \nu}=\sum_{n} \frac{2 i \omega \operatorname{Im} D_{\mu \nu}^{n}}{\omega_{n 0}^{2}-\omega^{2}},
\end{aligned}
$$

and for the negative frequencies $\omega<0$ we use the relation [27] $\alpha_{\mu \nu}(\omega)=\alpha_{\mu \nu}(-\omega)$. At the imaginary axes $\omega=i \xi$ it becomes real.

It was shown $[28,29]$ that, taking into account the atomic hyperfine structure, the polarizability tensor maybe decomposed in three contributions: scalar (diagonal), vector (antisymmetric), and tensor (symmetric and traceless). The sum of the scalar and tensor parts correspond to the symmetric part, $\alpha_{\mu \nu}^{s}$, and the vector one corresponds to the antisymmetric part, $\alpha_{\mu \nu}^{a}$, of polarizability (19). The antisymmetric part is defined by the total angular momentum, $\mathbf{J}$, of an atom [29,39]:

$$
\alpha_{\mu \nu}^{a}(\omega)=i \alpha_{v}(\omega) \epsilon_{\mu \nu \rho} J^{\rho},
$$

where $\epsilon_{\mu \nu \rho}$ is a completely antisymmetric tensor. The vector polarizability gives a contribution to the $P$-odd van der Waals forces [29] (see also review [30]).

The CP energy is obtained by the same rule (1) (see the Appendix and Ref. [40]). The CP energy reads

$$
\begin{aligned}
\mathcal{E}_{\mathrm{CP}}= & \iint \frac{d^{2} k}{4 \pi^{2}} \int_{0}^{\infty} \frac{d \xi}{\kappa} e^{-2 \kappa a} r_{a}^{i j} \\
& \times\left[\xi^{2} \alpha_{j i}+\alpha_{n i} k^{n} k_{j}+\alpha_{33} k_{i} k_{j}\right],
\end{aligned}
$$


where $\alpha_{i j}=\alpha_{i j}(i \xi)$ and $r_{a}^{i j}$ is the reflection matrix of the plane at $z=a$.

For a plane with arbitrary tensorial conductivity, the matrix $\mathbf{r}_{a}$ has form given by Eq. (3) and the CP energy becomes

$$
\begin{aligned}
\mathcal{E}_{\mathrm{CP}}(\alpha)= & -\iint \frac{d^{2} k}{(2 \pi)^{2}} \int_{0}^{\infty} \frac{d \xi}{\kappa} \\
& \times \frac{e^{-2 \kappa a}}{\xi^{2} \operatorname{tr} \boldsymbol{\eta}+\boldsymbol{k} \boldsymbol{k} \boldsymbol{\eta}+\kappa \xi(1+\operatorname{det} \boldsymbol{\eta})} \\
& \times\left\{\xi^{2}\left[\xi^{2} \operatorname{tr}(\boldsymbol{\eta} \boldsymbol{\alpha})+\boldsymbol{k} \boldsymbol{k}(\boldsymbol{\eta} \boldsymbol{\alpha}+\boldsymbol{\alpha} \boldsymbol{\eta})\right]\right. \\
& +(\boldsymbol{k} \boldsymbol{k} \boldsymbol{\eta})\left[(\boldsymbol{k} \boldsymbol{k} \boldsymbol{\alpha})+\alpha_{33} \kappa^{2}\right] \\
& \left.+\kappa \xi \operatorname{det} \boldsymbol{\eta}\left[\xi^{2} \operatorname{tr} \boldsymbol{\alpha}+(\boldsymbol{k} \boldsymbol{k} \boldsymbol{\alpha})+\alpha_{33} \boldsymbol{k}^{2}\right]\right\} .
\end{aligned}
$$

Here, $(\boldsymbol{k} \boldsymbol{k}(\boldsymbol{\eta} \boldsymbol{\alpha}))=k^{i} k^{j} \eta_{i n} \alpha_{n j}$ and $(\boldsymbol{k} \boldsymbol{k}(\boldsymbol{\alpha} \eta))=k^{i} k^{j} \alpha_{i n} \eta_{n j}$. We observe from this expression that the result does not depend on the components $\alpha_{3 i}$ and $\alpha_{i 3}$. This point was observed in Refs. [24]. We may rewrite the above expression in an invariant form by changing $\alpha_{33} \rightarrow \alpha_{\mu \nu} n^{\mu} n^{\nu}$, where vector $n^{\mu}$ is the unit vector perpendicular to the plane and assuming other $2 \mathrm{D}$ tensors living in this plane.

Accordingly, with the partition (19) we can represent the energy as sum of two contributions $\mathcal{E}_{\mathrm{CP}}=\mathcal{E}_{\mathrm{CP}}^{s}+\mathcal{E}_{\mathrm{CP}}^{a}$, where $\mathcal{E}_{\mathrm{CP}}^{s}=\mathcal{E}_{\mathrm{CP}}\left(\alpha^{s}\right), \mathcal{E}_{\mathrm{CP}}^{a}=\mathcal{E}_{\mathrm{CP}}\left(\alpha^{a}\right)$, and

$$
\mathcal{E}_{\mathrm{CP}}^{a}=-\iint \frac{d^{2} k}{(2 \pi)^{2}} \int_{0}^{\infty} \frac{\kappa \xi^{2} \operatorname{tr}\left(\boldsymbol{\eta} \boldsymbol{\alpha}^{a}\right) e^{-2 \kappa a} d \xi}{\xi^{2} \operatorname{tr} \boldsymbol{\eta}+\boldsymbol{k} \boldsymbol{k} \boldsymbol{\eta}+\kappa \xi(1+\operatorname{det} \boldsymbol{\eta})},
$$

is the contribution of the antisymmetric part of polarizability. In manifest form $\operatorname{tr}\left(\boldsymbol{\eta} \boldsymbol{\alpha}^{a}\right)=-\alpha_{12}^{a}\left(\eta_{12}-\eta_{21}\right)=$ $\widetilde{\alpha}_{v}(\xi) J_{z}\left(\eta_{12}-\eta_{21}\right)$, where $i \alpha_{v}(i \xi)=-\widetilde{\alpha}_{v}(\xi)$. This contribution is zero either for symmetric polarizability $\alpha$ or for the symmetric conductivity $\boldsymbol{\eta}$.

In the case of isotropic polarizability, $\alpha_{\mu \nu}=\alpha \delta_{\mu \nu}$, we obtain Eq. (7). In the case of anisotropic polarizability we can take a formal limit $\boldsymbol{\eta} \rightarrow \infty$ to consider a perfect metal plate, obtaining

$$
\begin{aligned}
\mathcal{E}_{\mathrm{CP}}^{i d}= & -\frac{1}{32 \pi a^{4}} \int_{0}^{\infty} d z e^{-z}\left\{\alpha_{\mu}^{\mu}\left(1+z+z^{2}\right)\right. \\
& \left.+\alpha_{33}\left(1+z-z^{2}\right)\right\},
\end{aligned}
$$

where $\alpha_{\mu \nu}=\alpha_{\mu \nu}(i z / 2 a)$. There is no contribution from the off-diagonal components. This expression is a generalization the CP energy for the isotropic molecule (8). For large distance, $a \rightarrow \infty$, we obtain

$$
\left.\mathcal{E}_{\mathrm{CP}}^{i d}\right|_{a \rightarrow \infty}=\mathcal{E}_{\mathrm{CP}}^{\infty}=-\frac{\alpha_{\mu}^{\mu}(0)}{8 \pi a^{4}},
$$

in accordance with Refs. [23,24]. It depends on the trace of the total polarizability tensor, and therefore far from the surface the CP energy does not depend on the orientation of the molecule.

For the conductivity tensor in the form (9) we obtain

$$
\begin{aligned}
& \mathcal{E}_{\mathrm{CP}}^{s}=-\iint_{0}^{\infty} \frac{k d k d \xi}{4 \pi \kappa} e^{-2 \kappa a} \frac{\left(\alpha_{11}+\alpha_{22}\right) a^{11}+\alpha_{33} a^{33}}{W} \\
& \mathcal{E}_{\mathrm{CP}}^{a}=\iint_{0}^{\infty} \frac{k d k d \xi}{\pi} e^{-2 \kappa a} \frac{Z \kappa \xi^{2} \alpha_{12}^{a}}{W}
\end{aligned}
$$

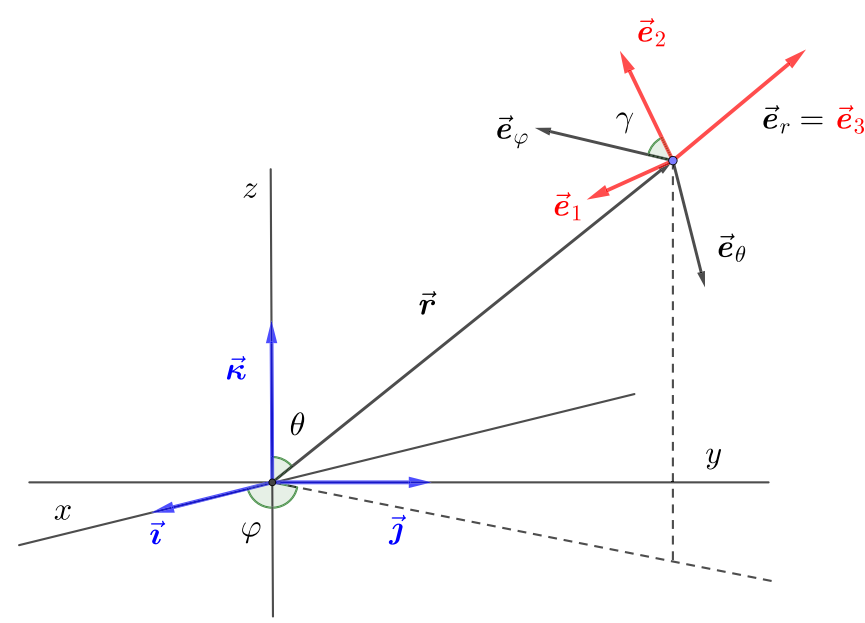

FIG. 2. The conductive plane is $(x, y)$, with a tensor conductivity $\sigma$. The eigenvectors of the polarizability tensor of a molecule are $\left(\vec{e}_{1}, \vec{e}_{2}, \vec{e}_{3}\right)$, which are obtained by rotation of the orthonormal spherical basis $\left(\vec{e}_{\theta}, \vec{e}_{\varphi}, \vec{e}_{r}\right)$ on the angle $\gamma$ around $\vec{e}_{r}$.

where $W$ is given in (11) and

$$
\begin{aligned}
& a^{11}=\kappa^{4}(X+Y)+\kappa \xi\left(\kappa^{2}+\xi^{2}\right)\left(X^{2}+X Y+Z^{2}\right)+\xi^{4} X, \\
& a^{33}=2 \kappa k^{2}\left[\kappa(X+Y)+\xi\left(X^{2}+X Y+Z^{2}\right)\right] .
\end{aligned}
$$

For pure constant Hall conductivity $X=Y=0$ one has

$$
\begin{aligned}
\mathcal{E}_{\mathrm{CP}}= & \frac{Z^{2}}{1+Z^{2}} \mathcal{E}_{\mathrm{CP}}^{i d} \\
& +\frac{Z}{4 \pi a^{4}\left(1+Z^{2}\right)} \int_{0}^{\infty} d z e^{-z} \alpha_{12}^{a}\left(\frac{i z}{2 a}\right) z(1+2 z),
\end{aligned}
$$

where $\mathcal{E}_{\mathrm{CP}}^{i d}$ is given by Eq. (23). The diagonal part of polarizability gives a contribution which is even with respect to conductivity $Z$, while the off-diagonal part is odd and it is zero for symmetric polarizability. The same result was obtained in Refs. [24] for CP interaction atom with Chern-Simons plane.

\section{CASIMIR TORQUE}

To calculate the Casimir torque we have to characterize the orientation in space of an anisotropically polarizable molecule. In Eq. (21) a molecule is situated in such way that it has polarizability tensor $\alpha_{\mu \nu}$ in the Cartesian coordinates $(x, y, z)$. Let us consider a symmetric tensor of the atomic polarizability, only. Suppose that the principal axes of the molecule are defined by the eigenvectors $\left(\vec{e}_{1}, \vec{e}_{2}, \vec{e}_{3}\right)$, which may be obtained by rotation the spherical basis $\left(\vec{e}_{\theta}, \vec{e}_{\varphi}, \vec{e}_{r}\right)$ on the angle $\gamma$ around $\vec{e}_{r}$ [23] (see Fig. 2):

$$
\begin{aligned}
& \vec{e}_{1}=\vec{e}_{\theta} \cos \gamma-\vec{e}_{\varphi} \sin \gamma, \\
& \vec{e}_{2}=\vec{e}_{\theta} \sin \gamma+\vec{e}_{\varphi} \cos \gamma, \\
& \vec{e}_{3}=\vec{e}_{r} .
\end{aligned}
$$

Note that setting $\vec{e}_{3}=\vec{e}_{r}$ is not restrictive, as we can always choose freely the origin of the coordinate system.

The matrix $\mathbf{T}$ of transformation from the old basis $(\vec{\imath}, \vec{\jmath}, \vec{k})$ to the new one $\left(\vec{e}_{1}, \vec{e}_{2}, \vec{e}_{3}\right)$ is defined by the relation

$$
\left(\vec{e}_{1} \vec{e}_{2} \vec{e}_{3}\right)=(\vec{\imath} \vec{j} \vec{k}) \cdot \mathbf{T} .
$$


The matrix $\mathbf{T}$ may easily be found from the relations (28). In a new basis, the polarization tensor has a diagonal form,

$$
\left[\alpha_{\mu \nu}\right]=\operatorname{diag}\left(\alpha_{11}, \alpha_{22}, \alpha_{33}\right)=\mathbf{T}^{T}\left[\alpha_{\mu \nu}^{\prime}\right] \mathbf{T} .
$$

Therefore, to calculate the energy we use the following representation of polarization tensor: $\left[\alpha_{\mu \nu}^{\prime}\right]=\mathbf{T}\left[\alpha_{\mu \nu}\right] \mathbf{T}^{T}$.

For $\theta=\varphi=\gamma=0$, i.e., when the principal axes of molecule are oriented along the Cartesian axes $(x, y, z)$, the old basis $(\vec{\imath}, \vec{\jmath}, \vec{k})$ coincides with new one $\left(\vec{e}_{1}, \vec{e}_{2}, \vec{e}_{3}\right)$ and the $\mathrm{CP}$ energy reads

$$
\mathcal{E}_{\mathrm{CP}}^{0}=-\iint \frac{d^{2} k}{(2 \pi)^{2}} \int_{0}^{\infty} \frac{d \xi}{\kappa} \frac{e^{-2 \kappa a} \alpha_{\mu \nu} A^{\mu \nu}}{\xi^{2} \operatorname{tr} \boldsymbol{\eta}+\boldsymbol{k} \boldsymbol{k} \boldsymbol{\eta}+\kappa \xi(1+\operatorname{det} \boldsymbol{\eta})},
$$

where

$$
\begin{aligned}
& A^{11}=\left(\boldsymbol{k} \boldsymbol{k} \boldsymbol{\eta}+\kappa \xi \operatorname{det} \boldsymbol{\eta}+\xi^{2} \operatorname{tr} \boldsymbol{\eta}\right)\left(\xi^{2}+k_{1}^{2}\right)-\xi^{2} \kappa^{2} \eta_{22}, \\
& A^{22}=\left(\boldsymbol{k} \boldsymbol{k} \boldsymbol{\eta}+\kappa \xi \operatorname{det} \boldsymbol{\eta}+\xi^{2} \operatorname{tr} \boldsymbol{\eta}\right)\left(\xi^{2}+k_{2}^{2}\right)-\xi^{2} \kappa^{2} \eta_{11}, \\
& A^{33}=\left(\kappa \boldsymbol{k} \boldsymbol{k} \boldsymbol{\eta}+\xi \boldsymbol{k}^{2} \operatorname{det} \boldsymbol{\eta}\right) \kappa .
\end{aligned}
$$

In the general case, $\theta \neq 0, \varphi \neq 0, \gamma \neq 0$, and we obtain the CP energy

$$
\mathcal{E}_{\mathrm{CP}}=\mathcal{E}_{\mathrm{CP}}^{0}+\Delta \mathcal{E}_{\mathrm{CP}}
$$

where

$$
\begin{aligned}
\Delta \mathcal{E}_{\mathrm{CP}}= & -\iint \frac{d^{2} k}{(2 \pi)^{2}} \int_{0}^{\infty} \frac{d \xi}{2 \kappa} \\
& \times \frac{e^{-2 \kappa a}}{\xi^{2} \operatorname{tr} \boldsymbol{\eta}+\boldsymbol{k} \boldsymbol{k} \boldsymbol{\eta}+\kappa \xi(1+\operatorname{det} \boldsymbol{\eta})} \\
& \times\left\{\left(\alpha_{11}-\alpha_{22}\right) S_{12}+\left(\alpha_{22}-\alpha_{33}\right) S_{23}\right\} .
\end{aligned}
$$

Here

$$
\begin{aligned}
S_{12}= & \cos 2 \gamma\left(B_{12} \sin 2 \varphi+B_{11} \cos 2 \varphi\right) \\
& -\cos \theta \sin 2 \gamma\left(B_{12} \cos 2 \varphi+B_{11} \sin 2 \varphi\right) \\
& -B_{11}+\cos ^{2} \gamma S_{23}, \\
S_{23}= & \sin ^{2} \theta\left\{k^{2}\left(\boldsymbol{k} \boldsymbol{k} \boldsymbol{\eta}+\kappa \xi \operatorname{det} \boldsymbol{\eta}+\xi^{2} \operatorname{tr} \boldsymbol{\eta}\right)\right. \\
& -\xi^{2} \kappa(2 \xi \operatorname{det} \boldsymbol{\eta}+\kappa \operatorname{tr} \boldsymbol{\eta}) \\
& \left.-B_{12} \sin 2 \varphi-B_{11} \cos 2 \varphi\right\},
\end{aligned}
$$

and

$$
\begin{aligned}
B_{11}= & \xi^{2} \kappa^{2}\left(\eta_{11}-\eta_{22}\right) \\
& +\left(k_{1}^{2}-k_{2}^{2}\right)\left(\boldsymbol{k} \boldsymbol{k} \boldsymbol{\eta}+\kappa \xi \operatorname{det} \boldsymbol{\eta}+\xi^{2} \operatorname{tr} \boldsymbol{\eta}\right), \\
B_{12}= & \xi^{2} \kappa^{2}\left(\eta_{12}+\eta_{21}\right)+2 k_{1} k_{2}\left(\boldsymbol{k} \boldsymbol{k} \boldsymbol{\eta}+\kappa \xi \operatorname{det} \boldsymbol{\eta}+\xi^{2} \operatorname{tr} \boldsymbol{\eta}\right) .
\end{aligned}
$$

In fact, the expression (32) for energy is another representation of Eq. (21) with explicit indication of the orientation of the molecule. For isotropic molecules with $\alpha_{\mu \nu}=\alpha \delta_{\mu \nu}$ the energy correction $\Delta \mathcal{E}_{\mathrm{CP}}=0$, as should be the case.

Let us consider the general form of conductivity tensor (9). We use polar coordinates for $\left(k_{1}, k_{2}\right)$ and after integrating over angular variables we obtain that $\mathcal{E}_{\mathrm{CP}}^{0}=\mathcal{E}_{\mathrm{CP}}^{s}$, where $\mathcal{E}_{\mathrm{CP}}^{s}$ is given by Eq. (25), and

$$
\Delta \mathcal{E}_{\mathrm{CP}}=-\sin ^{2} \theta\left(\mathcal{G}_{12} \cos ^{2} \gamma+\mathcal{G}_{23}\right)
$$

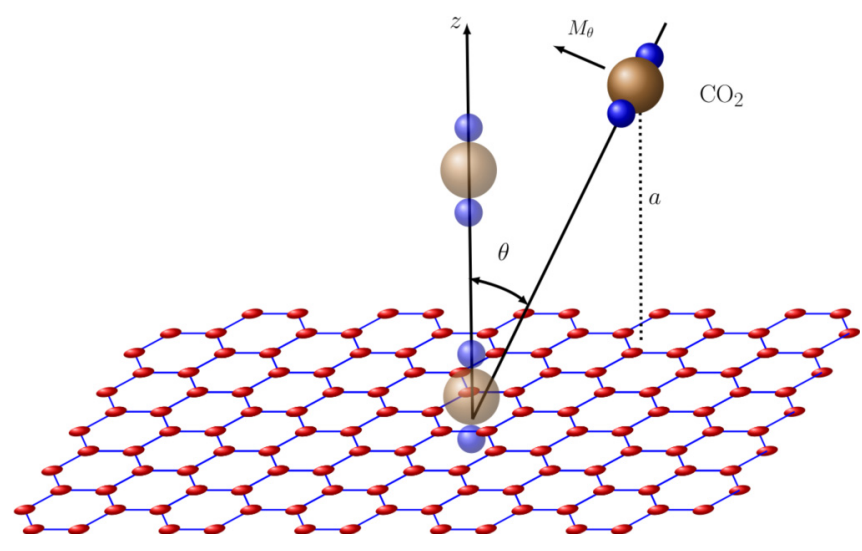

FIG. 3. Schematic figure showing the arrangement of the molecule $\mathrm{CO}_{2}$ and the plane. The Casimir torque $M_{\theta}$ tends to rotate molecules to the direction of the axis $z$ and as the result molecules can go through the membrane.

where

$$
\begin{aligned}
\mathcal{G}_{i j}= & \iint_{0}^{\infty} \frac{k d k d \xi}{4 \pi \kappa} e^{-2 \kappa a} \frac{\alpha_{i i}-\alpha_{j j}}{W}\left\{\kappa^{2}(X+Y)\left(\kappa^{2}-2 \xi^{2}\right)\right. \\
& \left.+\kappa \xi\left(\kappa^{2}-3 \xi^{2}\right)\left(X^{2}+X Y+Z^{2}\right)-X \xi^{4}\right\} .
\end{aligned}
$$

The energy has no dependence on the angle $\varphi$ due to isotropy of the conductivity plane. The angle $\gamma$ gives a contribution in the case $\alpha_{11} \neq \alpha_{22}$, only.

In general, the $\mathrm{CP}$ energy depends on three angles $(\theta, \varphi, \gamma)$ which define orientation of the molecule in the space. Therefore, we may define the Casimir torque in relation to each angle [25]:

$$
\begin{aligned}
& M_{\theta}=-\partial_{\theta} \mathcal{E}_{\mathrm{CP}}=\sin 2 \theta\left(\mathcal{G}_{12} \cos ^{2} \gamma+\mathcal{G}_{23}\right), \\
& M_{\varphi}=-\partial_{\varphi} \mathcal{E}_{\mathrm{CP}}=0, \\
& M_{\gamma}=-\partial_{\gamma} \mathcal{E}_{\mathrm{CP}}=\sin ^{2} \theta \sin 2 \gamma \mathcal{G}_{12} .
\end{aligned}
$$

Zero torque with minimum energy $\mathcal{E}_{\mathrm{CP}}$ describes the equilibrium states.

\section{The molecule $\mathrm{CO}_{2}$}

The molecule $\mathrm{CO}_{2}$ (see Fig. 3) has anisotropic polarizability and $\alpha_{11}=\alpha_{22} \neq \alpha_{33}$ (see Fig. 4), and for the whole interval of the imaginary frequencies the inequality $\alpha_{33}>\alpha_{22}$ holds. The CP energy depends on $\theta$ only,

$$
\mathcal{E}_{\mathrm{CP}}=\mathcal{E}_{\mathrm{CP}}^{0}-\sin ^{2} \theta \mathcal{G}_{23},
$$

where $\mathcal{E}_{\mathrm{CP}}^{0}=\mathcal{E}_{\mathrm{CP}}^{s}$ and $\mathcal{G}_{23}$ are given by Eqs. (25) and (36), respectively. The $\sin ^{2} \theta$ dependence has already been observed for a dielectric slab in Ref. [23].

In this case only $M_{\theta}$ remains nonzero, $M_{\theta}=\sin 2 \theta G_{23}$. The $2 \theta$ dependence was also observed in Ref. [25]. Zero torque, $M=0$, which corresponds to extrema of the energy as a function of the angle, is realized at the angles $\theta_{e}=\left(0, \frac{\pi}{2}, \pi\right)$ with energies $\mathcal{E}_{\mathrm{CP}}=\left(\mathcal{E}_{\mathrm{CP}}^{0}, \mathcal{E}_{\mathrm{CP}}^{0}-\mathcal{G}_{23}, \mathcal{E}_{\mathrm{CP}}^{0}\right)$. The angles $\theta=$ $0, \pi$ correspond to positions perpendicular to the surface and $\theta=\pi / 2$ corresponds to a position parallel to the surface. Let us consider some specific situations. 


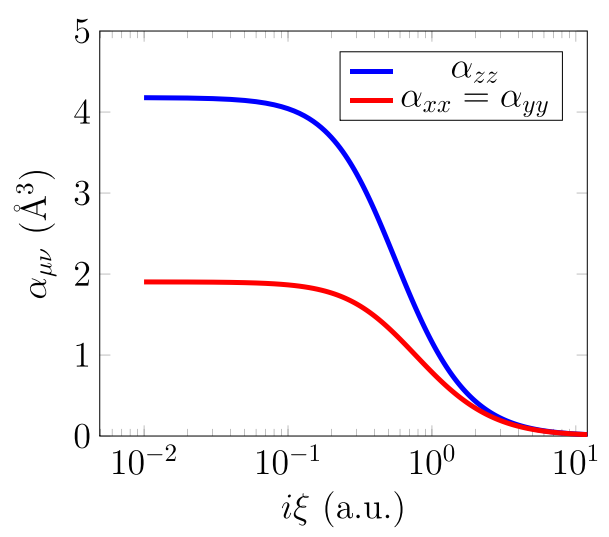

FIG. 4. The polarizability of $\mathrm{CO}_{2}$. The numerical data were provided by the authors of Ref. [23]. The imaginary frequency $\xi$ is measured in a.u. and polarizability is measured in $\AA^{3}$. Here 1 a.u. $=4.13413 \times 10^{16} \mathrm{rad} / \mathrm{s}=27.212 \mathrm{eV}$.

\section{Constant Hall conductivity}

For pure Hall constant conductivity we set $X=Y=0$ and we obtain from (32)

$$
\begin{aligned}
\mathcal{E}_{\mathrm{CP}}^{0}= & -\frac{Z^{2}}{1+Z^{2}} \int_{0}^{\infty} \frac{d y y^{3}}{2 \pi a^{4}} \int_{0}^{1} d x e^{-2 y} \\
& \times\left[\left(1+x^{2}\right) \alpha_{22}+\left(1-x^{2}\right) \alpha_{33}\right], \\
\mathcal{G}_{23}= & \frac{Z^{2}}{1+Z^{2}} \int_{0}^{\infty} \frac{d y y^{3}}{4 \pi a^{4}} \int_{0}^{1} d x e^{-2 y} \\
& \times\left(1-3 x^{2}\right)\left(\alpha_{22}-\alpha_{33}\right),
\end{aligned}
$$

where $\alpha_{i j}=\alpha_{i j}\left(\frac{i x y}{a}\right)$.

For $a \rightarrow \infty$ we obtain [see (24)]

$$
\begin{aligned}
\mathcal{E}_{\mathrm{CP}}^{0} & \rightarrow \mathcal{E}_{\mathrm{CP}}^{\infty}=-\frac{\alpha_{\mu}^{\mu}(0)}{8 \pi a^{4}}, \\
\mathcal{G}_{23} & =O\left(a^{-5}\right) .
\end{aligned}
$$

For numerical evaluation we normalize the energy on its asymptotic value $\left|\mathcal{E}_{\mathrm{CP}}^{\infty}\right|=\frac{\alpha_{\mu}^{\mu}(0)}{8 \pi a^{4}}: \mathcal{E}_{\mathrm{CP}} /\left|\mathcal{E}_{\mathrm{CP}}^{\infty}\right|=\frac{Z^{2}}{1+Z^{2}} E_{\mathrm{CP}}$ and $\mathcal{G}_{23} /\left|\mathcal{E}_{\mathrm{CP}}^{\infty}\right|=\frac{Z^{2}}{1+Z^{2}} G_{23}$. The plots of the whole energy $E_{\mathrm{CP}}$ and separately the $G_{23}$ contribution, responsible for the $\theta$ dependence, are shown in Fig. 5. We observe that the $\mathrm{CP}$
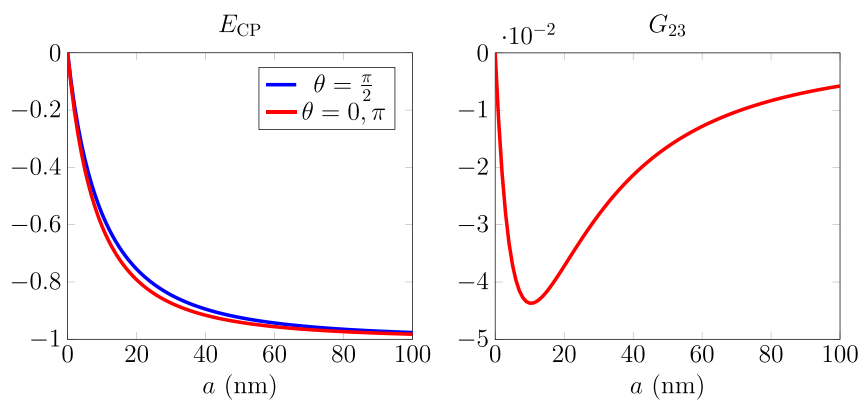

FIG. 5. Left panel: the dimensionless energy $E_{\mathrm{CP}}$ for extremal angles. The energy minima are realized for $\theta=0, \pi$. Right panel: $G_{23}$ contribution (dimensionless), responsible for the angle dependence. It is always negative. energy with angles $\theta=0, \pi$ is smaller than the energy for $\theta=\pi / 2$ for any distance between atom and plane. It means that equilibrium positions of molecules are perpendicular to the plane.

\section{Graphene}

In this case $X=\eta_{\mathrm{te}}, Y=\eta_{\mathrm{tm}}-\eta_{\mathrm{te}}$, and $Z=0$, where $\eta_{\mathrm{te}}$ and $\eta_{\mathrm{tm}}$ are conductivities of TE and TM modes, respectively [37]. The energy for zero temperature reads

$$
\begin{aligned}
\mathcal{E}_{\mathrm{CP}}^{0}= & -\int_{0}^{\infty} \frac{k d k}{2 \pi} \int_{0}^{\infty} \frac{d \xi}{\kappa} e^{-2 \kappa a} \\
& \times\left\{-\alpha_{22} \xi^{2} r_{\mathrm{te}}+\left[\alpha_{22} \kappa^{2}+\alpha_{33}\left(\kappa^{2}-\xi^{2}\right)\right] r_{\mathrm{tm}}\right\} \\
\mathcal{G}_{23}= & \int_{0}^{\infty} \frac{k d k}{4 \pi} \int_{0}^{\infty} \frac{d \xi}{\kappa} e^{-2 \kappa a}\left(\alpha_{22}-\alpha_{33}\right) \\
& \times\left[\xi^{2} r_{\mathrm{te}}+\left(\kappa^{2}-2 \xi^{2}\right) r_{\mathrm{tm}}\right]
\end{aligned}
$$

where

$$
r_{\mathrm{te}}=-\frac{\eta_{\mathrm{te}}}{\eta_{\mathrm{te}}+\frac{\kappa}{\xi}}, \quad r_{\mathrm{tm}}=\frac{\eta_{\mathrm{tm}}}{\eta_{\mathrm{tm}}+\frac{\xi}{\kappa}},
$$

are reflection coefficients for graphene and $\eta_{\mathrm{te}, \mathrm{tm}}=2 \pi \sigma_{\mathrm{te}, \mathrm{tm}}$. At zero temperature, the energy can also depend, in general, on the chemical potential $\mu$ [13]. In the gapeless case the conductivities of TM and TE modes have the following form [37]:

$$
\begin{aligned}
& \frac{\eta_{\mathrm{tm}}}{\eta_{\mathrm{gr}}}=\frac{\xi}{k_{F}}+\frac{8}{\pi k_{F}} \operatorname{Re} \int_{0}^{|\mu|} d z \frac{q r-\xi k_{F}}{k_{F} r+q \xi}, \\
& \frac{\eta_{\mathrm{te}}}{\eta_{\mathrm{gr}}}=\frac{k_{F}}{\xi}+\frac{8}{\pi \xi} \operatorname{Re} \int_{0}^{|\mu|} d z \frac{q\left(q^{2}-\xi^{2}\right)}{r\left(q k_{F}+\xi r\right)},
\end{aligned}
$$

where $k_{F}=\sqrt{\xi^{2}+v_{F}^{2} k^{2}}, q=\xi-i z, r=\sqrt{q^{2}+v_{F}^{2} k^{2}}$, and $\eta_{\mathrm{gr}}=2 \pi e^{2} / 4$ is the universal conductivity of graphene.

The nonzero temperature maybe included by changing $\int_{0}^{\infty} d \xi \rightarrow 2 \pi T \sum_{n=0}^{\prime \infty}$ and $\xi \rightarrow \xi_{n}$, where $\xi_{n}=2 \pi n T$ are the Matsubara frequencies:

$$
\begin{aligned}
\mathcal{E}_{\mathrm{CP}}^{0}= & -T \sum_{n=0}^{\infty} \int_{0}^{\infty} \frac{k d k}{\kappa_{n}} e^{-2 \kappa_{n} a} \\
& \times\left\{-\alpha_{22}^{n} \xi_{n}^{2} r_{\mathrm{te}}^{n}+\left[\alpha_{22}^{n} \kappa_{n}^{2}+\alpha_{33}^{n}\left(\kappa_{n}^{2}-\xi_{n}^{2}\right)\right] r_{\mathrm{tm}}^{n}\right\}, \\
\mathcal{G}_{23}= & T \sum_{n=0}^{\infty} \int_{0}^{\infty} \frac{k d k}{2 \kappa_{n}} e^{-2 \kappa_{n} a}\left(\alpha_{22}^{n}-\alpha_{33}^{n}\right) \\
& \times\left[\xi_{n}^{2} r_{\mathrm{te}}^{n}+\left(\kappa_{n}^{2}-2 \xi_{n}^{2}\right) r_{\mathrm{tm}}^{n}\right],
\end{aligned}
$$

where $\kappa_{n}=\sqrt{k^{2}+\xi_{n}^{2}}$ and $\alpha_{\mu \nu}^{n}=\alpha_{\mu \nu}\left(i \xi_{n}\right)$. The conductivities of the graphene in this case read

$$
\begin{aligned}
& \frac{\eta_{\mathrm{tm}}}{\eta_{\mathrm{gr}}}=\frac{\xi}{k_{F}}+\frac{8}{\pi k_{F}} \operatorname{Re} \int_{0}^{\infty} d z \frac{q r-\xi k_{F}}{k_{F} r+q \xi} \Theta(z), \\
& \frac{\eta_{\mathrm{te}}}{\eta_{\mathrm{gr}}}=\frac{k_{F}}{\xi}+\frac{8}{\pi \xi} \operatorname{Re} \int_{0}^{\infty} d z \frac{q\left(q^{2}-\xi^{2}\right)}{r\left(q k_{F}+\xi r\right)} \Theta(z),
\end{aligned}
$$

where

$$
\Theta(z)=\frac{1}{e^{\frac{z+\mu}{T}}+1}+\frac{1}{e^{\frac{z-\mu}{T}}+1}
$$



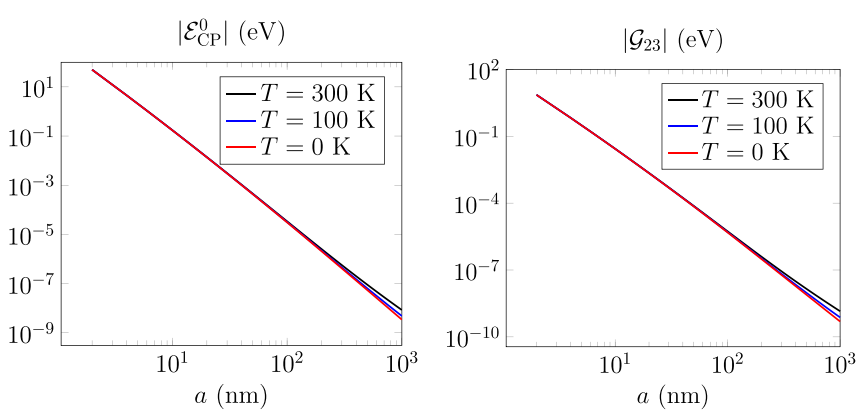

FIG. 6. Left panel: the energy $\left|\mathcal{E}_{\mathrm{CP}}^{0}\right|$ in double log scale for different temperatures. Right panel: the torque amplitude $\left|\mathcal{G}_{\mathrm{CP}}\right|$ in double $\log$ format for various temperatures. For small distances the energies $\sim 1 / a^{4}$. For great distance and nonzero temperatures the energies $\sim T / a^{3}$. The mass gap and the chemical potential are zero, $m=\mu=0$.

Let us consider the asymptotic $a \rightarrow \infty$. In the case of zero temperature we change variables $k \rightarrow k / a, \xi \rightarrow \xi / a$ in (41) and take the formal limit $a \rightarrow \infty$. In this limit we obtain

$$
\begin{aligned}
& \frac{\eta_{\mathrm{tm}}}{\eta_{\mathrm{gr}}} \rightarrow \frac{8 a|\mu| \xi\left(k_{F}+\xi\right)}{\pi k^{2} k_{F} v_{F}^{2}} \rightarrow \infty, \\
& \frac{\eta_{\mathrm{te}}}{\eta_{\mathrm{gr}}} \rightarrow-\frac{8 a|\mu| \xi\left(k_{F}+\xi\right)}{\pi k^{2} k_{F} v_{F}^{2}} \rightarrow-\infty .
\end{aligned}
$$

The reflection coefficients become those of a perfect metal, $r_{\mathrm{tm}}=1$ and $r_{\mathrm{te}}=-1$, and

$$
\mathcal{E}_{\mathrm{CP}}^{0} / \mathcal{E}_{\mathrm{CP}}^{\infty} \rightarrow 1, \quad \mathcal{G}_{23} / \mathcal{E}_{\mathrm{CP}}^{\infty} \rightarrow 0 .
$$

This regime is realized in the case $a|\mu| \gg 1$. If $\mu=0$, the conductivities are given by the first terms in Eq. (45) and $r_{\mathrm{tm}} \neq 1$ and $r_{\mathrm{te}} \neq-1$. Numerically, we obtain

$$
\begin{aligned}
& \mathcal{E}_{\mathrm{CP}}^{0} / \mathcal{E}_{\mathrm{CP}}^{\infty} \rightarrow \frac{\alpha_{22}(0) I_{1}+\alpha_{33}(0) I_{2}}{\alpha_{v}^{v}(0)} \approx 0.0542, \\
& \mathcal{G}_{23} / \mathcal{E}_{\mathrm{CP}}^{\infty} \rightarrow-\frac{\alpha_{22}(0)-\alpha_{33}(0)}{\alpha_{v}^{v}(0)} I_{3} \approx 0.0077,
\end{aligned}
$$

where $I_{1} \approx 0.079, I_{2} \approx 0.067$, and $I_{3} \approx 0.027$.

In the case of nonzero temperature, the main contribution comes from zero term of sum in (44):

$$
\begin{aligned}
& \mathcal{E}_{\mathrm{CP}}^{0} / \mathcal{E}_{\mathrm{CP}}^{\infty} \rightarrow \pi a T \frac{\alpha_{22}(0)+\alpha_{33}(0)}{\alpha_{v}^{\nu}(0)} \approx \frac{2 \pi a T}{3} 1.14, \\
& \mathcal{G}_{23} / \mathcal{E}_{\mathrm{CP}}^{\infty} \rightarrow-\pi a T \frac{\alpha_{22}(0)-\alpha_{33}(0)}{2 \alpha_{\nu}^{\nu}(0)} \approx \frac{2 \pi a T}{3} 0.21 .
\end{aligned}
$$

The numerical analysis reveals that the function $\mathcal{G}_{23}$ is negative for any distance between plane and molecule. It means that the energy minima are realized for $\theta=0, \pi$. The module of energies are shown in Fig. 6 in double log scale in the range $a \in[1,500] \mathrm{nm}$ and for temperatures $T=0,100,300 \mathrm{~K}$. We observe that for zero temperature the energies $\mathcal{E}_{\mathrm{CP}}^{0}$ and $\mathcal{G}_{23}$ are proportional to $a^{-4}$. The nonzero temperature changes behavior of the $\mathrm{CP}$ energy at large distance between graphene and molecule which becomes proportional to $T / a^{3}$ according to $(50)$.

There are two comments to be made. First, we consider here the case $\mu=0$. It would be interesting to investigate how the combined effects of the nonzero band gap and chemical potential [41] might modify the Casimir-Polder force for anisotropic particles. Second, it would be interesting to study how the $\mathrm{CP}$ energy and torque at very small separations (corresponding to virtual energies of above $3 \mathrm{eV}$ ) might be influenced by the full band structure of graphene, which is not entirely taken into account by the model of Ref. [37]. These questions are beyond the scope of this paper and deserve further investigations.

The energy for extremal angles $\theta=0, \pi / 2, \pi$ and for temperatures $T=0,100,300 \mathrm{~K}$ are shown in Fig. 7. In all situations the minima of energy are realized for $\theta=0, \pi$, when the molecule is perpendicular to the surface (see Fig. 3).

\section{SUMMARY AND CONCLUSION}

We considered the Casimir-Polder energy for an anisotropic molecule near a conductive plane with tensorial surface conductivity. The CP energy is a sum of the contributions from symmetric and antisymmetric (22) parts of polarizability. The latter is zero either for the symmetric polarizability tensor or for the symmetric conductivity tensor. The CP energy is represented in the form with manifest dependence on the orientation of the molecule (32). The Casimir torque is given by derivatives with respect to angles of orientation of the molecule (38). The energy has no dependence on the spherical angle $\varphi$ due to the freedom of choosing the origin of coordinates, and dependence on $\theta$ is of the type $\sin ^{2} \theta$. The dependence on the angle $\gamma$ is only present in the case $\alpha_{11} \neq \alpha_{22}$. This angle characterizes a rotation around a radius vector to the molecule (see Fig. 2).

The molecule $\mathrm{CO}_{2}$ has tensor polarizability with $\alpha_{11}=$ $\alpha_{22} \neq \alpha_{33}$ and $\alpha_{33}>\alpha_{11}$ for any frequency. For this reason the energy depends on $\theta$ as $\sin ^{2} \theta$ and the minima of the energy and zero torque are realized for $\theta=0, \pi$ when the molecule is perpendicular to the surface, and the maximum is realized for $\theta=\pi / 2$. The same result has been obtained for the molecule $\mathrm{CO}_{2}$ and a dielectric slab in Ref. [23]. Numerical results for graphene and the molecule $\mathrm{CO}_{2}$ are shown in Fig. 7 for different temperatures.

Separating $\mathrm{CO}_{2}$ from the atmosphere is a pressing technological issue, in many cases addressed with usage of graphene and graphene oxide membranes [42,43], including those of single-layered graphene [44]. Our study revealed that the Casimir torque acting on the molecules tends to shift them into a position favoring easier penetration through such membranes, with the smallest cross-section side facing the interface. In the studied cases, see Fig. 8, the torque is shown to be enhanced by enlarging the Hall conductivity contribution of the conductivity tensor of the membrane, which is notably pronounced in the case of graphene. It permits us to conjecture that creating membranes with larger antisymmetric conductivities might benefit $\mathrm{CO}_{2}$ separation technologies.

\section{ACKNOWLEDGMENTS}

N.K. was supported in part by Grants No. 2019/10719-9 and No. 2016/03319-6 of the São Paulo Research Foundation (FAPESP) and by the Russian Foundation for Basic Research Grant No. 19-02-00496-a. One of us (N.K.) is grateful 

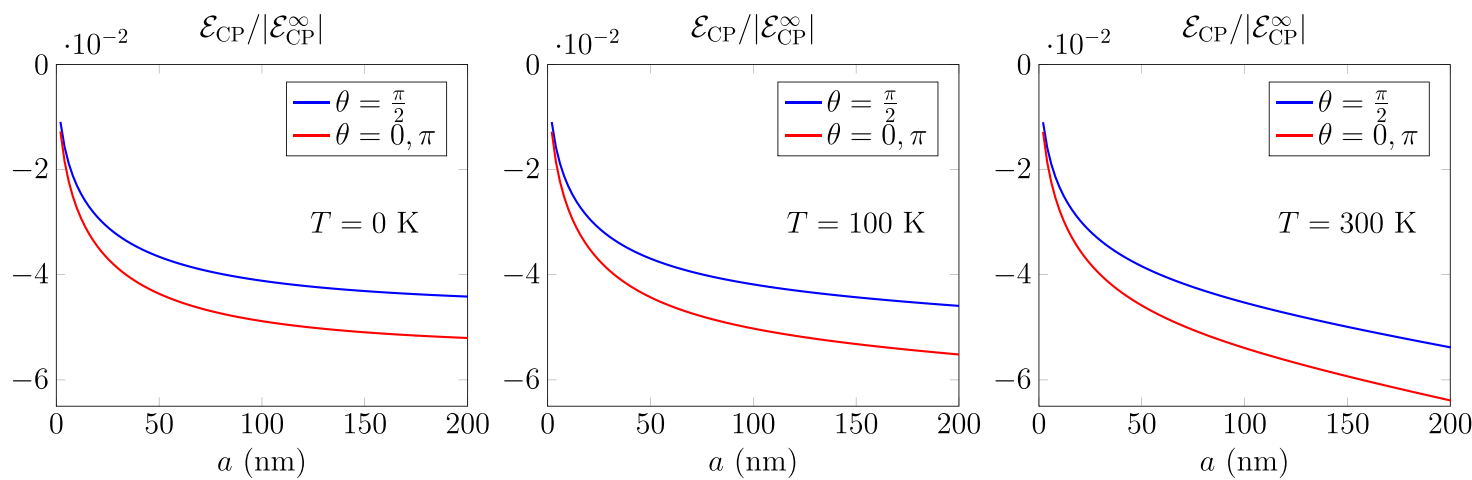

FIG. 7. The energy $\mathcal{E}_{\mathrm{CP}} /\left|\mathcal{E}_{\mathrm{CP}}^{\infty}\right|$ for temperature $T=0 \mathrm{~K}$ (left panel), $T=100 \mathrm{~K}$ (middle panel), and $T=300 \mathrm{~K}$ (right panel). The minima of the energy are realized for $\theta=0, \pi$.

to Valery Marachevsky for fruitful discussions. The authors thank the authors of the Ref. [23] for numerical data on the polarizability tensor of the molecule $\mathrm{CO}_{2}$.

\section{APPENDIX: DERIVATION OF CP ENERGY}

\section{Eigenproblem}

The Maxwell equations in anisotropic media give a dispersion relation which has, in general, four distinctive roots [see below in (A6)] $k_{3}=k_{3}\left(k_{1}, k_{2}, \omega\right)$ and four corresponding distinct amplitudes $\mathcal{E}_{n}$ and $\mathcal{H}_{n}(n=1,2,3,4)$. To obtain this dispersion relation we search for solutions of the Maxwell equations in the plane-waves form

$$
\mathbf{E}=e^{i k_{1} x+i k_{2} y+i k_{3} z-i \omega t} \mathcal{E}, \quad \mathbf{H}=e^{i k_{1} x+i k_{2} y+i k_{3} z-i \omega t} \mathcal{H},
$$

with constant amplitudes $\mathcal{E}$ and $\mathcal{H}$.

The equations can be represented in the form of an eigenproblem,

$$
\mathbf{M} \cdot \boldsymbol{v}=k_{3} \boldsymbol{v},
$$

where the matrix $\mathbf{M}$ is given by

$$
\mathbf{M}=\left[\begin{array}{cccc}
-k_{1} \frac{\varepsilon_{31}}{\varepsilon_{33}} & -k_{1} \frac{\varepsilon_{32}}{\varepsilon_{33}} & \frac{k_{1} k_{2}}{\omega \varepsilon_{33}} & \omega-\frac{k_{1}^{2}}{\omega \varepsilon_{33}} \\
-k_{2} \frac{\varepsilon_{31}}{\varepsilon_{33}} & -k_{2} \frac{\varepsilon_{32}}{\varepsilon_{33}} & -\omega+\frac{k_{2}^{2}}{\omega \varepsilon_{33}} & -\frac{k_{1} k_{2}}{\omega \varepsilon_{33}} \\
-\frac{k_{1} k_{2}}{\omega}-\frac{\omega e_{12}}{\varepsilon_{33}} & \frac{k_{1}^{2}}{\omega}-\frac{\omega e_{11}}{\varepsilon_{33}} & -k_{2} \frac{\varepsilon_{23}}{\varepsilon_{33}} & k_{1} \frac{\varepsilon_{23}}{\varepsilon_{33}} \\
-\frac{k_{2}^{2}}{\omega}+\frac{\omega e_{22}}{\varepsilon_{33}} & \frac{k_{1} k_{2}}{\omega}+\frac{\omega e_{21}}{\varepsilon_{33}} & k_{2} \frac{\varepsilon_{13}}{\varepsilon_{33}} & -k_{1} \frac{\varepsilon_{13}}{\varepsilon_{33}}
\end{array}\right],
$$
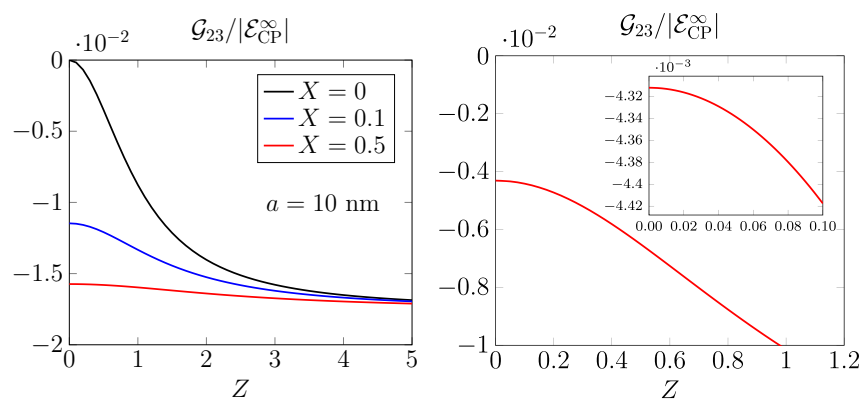

FIG. 8. The dependence of the torque amplitude $\mathcal{G}_{23}$ on the antisymmetric (Hall) part, $Z$, of the conductivity in the case of constant symmetric conductivity (left panel) and for graphene (right panel). and the four-component "vector" $v$ reads

$$
\boldsymbol{v}=\left(\begin{array}{c}
\mathcal{E}_{x} \\
\mathcal{E}_{y} \\
\mathcal{H}_{x} \\
\mathcal{H}_{y}
\end{array}\right) \equiv\left(\begin{array}{l}
\boldsymbol{e} \\
\boldsymbol{h}
\end{array}\right),
$$

which contains the tangent components of the electromagnetic field. Here, $e_{\mu \nu}$ is a matrix of minors of the elements of $\varepsilon_{\mu \nu}$. To find a solution we need only these components of the electromagnetic field, since the third components are readily obtained as

$$
\begin{aligned}
\mathcal{E}_{z} & =\frac{1}{\omega \varepsilon_{33}}\left[k_{2} \mathcal{H}_{x}-k_{2} \mathcal{H}_{y}-\omega\left(\varepsilon_{31} \mathcal{E}_{x}+\varepsilon_{32} \mathcal{E}_{y}\right)\right], \\
\mathcal{H}_{z} & =\frac{1}{\omega}\left(k_{1} \mathcal{E}_{y}-k_{2} \mathcal{E}_{x}\right) .
\end{aligned}
$$

The dispersion relation, $k_{3}=k_{3}(\boldsymbol{k}, \omega)$, is solution of the fourth-degree equation

$$
\varepsilon_{33} \operatorname{det}\left(\mathbf{M}-k_{3} \mathbf{I}\right) \equiv \varepsilon_{33} k_{3}^{4}+a_{1} k_{3}^{3}+a_{2} k_{3}^{2}+a_{3} k_{3}+a_{4}=0,
$$

where coefficients

$$
\begin{aligned}
a_{1}= & k^{i}\left(\varepsilon_{i 3}+\varepsilon_{3 i}\right), \\
a_{2}= & k^{2} \varepsilon_{33}+\boldsymbol{k} \boldsymbol{k}-\omega^{2}\left(e_{11}+e_{22}\right), \\
a_{3}= & \boldsymbol{k}^{2} k^{i}\left(\varepsilon_{i 3}+\varepsilon_{3 i}\right)+\omega^{2} k^{i}\left(\varepsilon_{i 3}^{-1}+\varepsilon_{3 i}^{-1}\right) \operatorname{det} \boldsymbol{\varepsilon}, \\
a_{4}= & \boldsymbol{k}^{2} \boldsymbol{k} \boldsymbol{k} \boldsymbol{\varepsilon}-\omega^{2}\left(\boldsymbol{k}^{2} e_{33}+\varepsilon_{33} \boldsymbol{k} \boldsymbol{k}-k^{i} \varepsilon_{i 3} k^{j} \varepsilon_{3 j}\right) \\
& +\omega^{4} \operatorname{det} \boldsymbol{\varepsilon},
\end{aligned}
$$

are invariants over rotation in plane $z=0$. We denote the roots of (A6) as $\kappa_{n}$. We choose enumeration of roots such that in the case of vacuum $\kappa_{1,2} \rightarrow+k_{z}$ and $\kappa_{3,4} \rightarrow-k_{z}$, where $k_{z}=$ $\sqrt{\omega^{2}-\boldsymbol{k}^{2}}$.

In the case of vacuum, $\varepsilon_{\mu \nu}=\delta_{\mu \nu}$, the dispersion relation has double-degenerate roots $\kappa_{1,2}=+k_{z}$ and $\kappa_{3,4}=-k_{z}$. Corresponding eigenvectors read

$$
\boldsymbol{v}_{1}^{r}=\left(\begin{array}{c}
1 \\
0 \\
-\frac{k_{1} k_{2}}{\omega k_{z}} \\
\frac{k_{1}^{2}+k_{z}^{2}}{\omega k_{z}}
\end{array}\right), \quad \boldsymbol{v}_{2}^{r}=\left(\begin{array}{c}
0 \\
1 \\
-\frac{k_{2}^{2}+k_{z}^{2}}{\omega k_{z}} \\
\frac{k_{1} k_{2}}{\omega k_{z}}
\end{array}\right),
$$

and $\left(\boldsymbol{v}_{3}^{r}, \boldsymbol{v}_{4}^{r}\right)=\left(\boldsymbol{v}_{3}^{r}, \boldsymbol{v}_{4}^{r}\right)_{k_{z} \rightarrow-k_{z}}$. 
Then, the general form of the field is a linear combination of these solutions:

$$
\boldsymbol{v}^{r}=e^{i k_{z} z} v_{1}^{r} \boldsymbol{v}_{1}^{r}+e^{i k_{z} z} v_{2}^{r} \boldsymbol{v}_{2}^{r}+e^{-i k_{z} z} v_{3}^{r} \boldsymbol{v}_{3}^{r}+e^{-i k_{z} z} v_{4}^{r} \boldsymbol{v}_{4}^{r},
$$

with common factor $e^{i k_{1} x+i k_{2} y-i \omega t}$.

In the nonvacuum case the amplitudes read

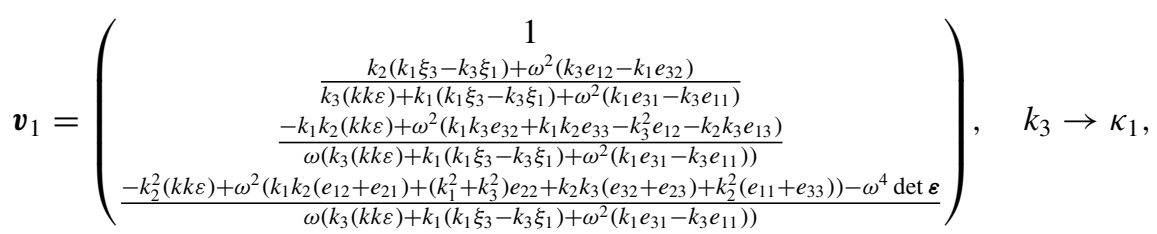

$$
\begin{aligned}
& \boldsymbol{v}_{2}=\left(\begin{array}{c}
\frac{k_{1}\left(k_{2} \xi_{3}-k_{3} \xi_{2}\right)+\omega^{2}\left(k_{3} e_{21}+k_{2} e_{31}\right)}{k_{3}(k k \varepsilon)+k_{2}\left(k_{2} \xi_{3}-k_{3} \xi_{2}\right)-\omega^{2}\left(k_{2} e_{32}+k_{3} e_{22}\right)} \\
1 \\
\frac{k_{1}^{2}(k k \varepsilon)+\omega^{2}\left(-k_{1} k_{2}\left(e_{12}+e_{21}\right)-\left(k_{2}^{2}+k_{3}^{2}\right) e_{11}+k_{1} k_{3}\left(e_{31}+e_{13}\right)-k_{1}^{2}\left(e_{22}+e_{33}\right)\right)+\omega^{4} \operatorname{det} \varepsilon}{\omega\left(k_{3}(k k \varepsilon)+k_{2}\left(k_{2} \xi_{3}-k_{3} \xi_{2}\right)-\omega^{2}\left(k_{2} e_{32}+k_{3}(2)\right)\right)} \\
\frac{k_{1} k_{2}(k k \varepsilon)+\omega^{2}\left(-k_{1} k_{3} e_{23}-k_{1} k_{2} e_{33}+k_{3}^{2} e_{21}+k_{2} k_{3} 3_{31}\right)}{\omega\left(k_{3}(k k \varepsilon)+k_{2}\left(k_{2} \xi_{3}-k_{3} \xi_{2}\right)-\omega^{2}\left(k_{2} e_{32}+k_{3} e_{22}\right)\right)}
\end{array}\right), \quad k_{3} \rightarrow \kappa_{2},
\end{aligned}
$$

where $e_{\mu \nu}$, as before, is a matrix of minors of $\boldsymbol{\varepsilon}_{\mu \nu}, \xi_{\nu}=k^{\mu} \varepsilon_{\mu \nu}$, and $(k k \varepsilon)=k^{\mu} k^{v} \varepsilon_{\mu \nu}$. Also $\boldsymbol{v}_{3}=\left.\boldsymbol{v}_{1}\right|_{k_{3} \rightarrow \kappa_{3}}, \boldsymbol{v}_{4}=\left.\boldsymbol{v}_{2}\right|_{k_{3} \rightarrow \kappa_{4}}$.

The general solution is a linear combination of these four solutions:

$$
\boldsymbol{v}=e^{i \kappa_{1} z} v_{1}^{l} \boldsymbol{v}_{1}+e^{i \kappa_{2} z} v_{2}^{l} \boldsymbol{v}_{2}+e^{i \kappa_{3} z} v_{3}^{l} \boldsymbol{v}_{3}+e^{i \kappa_{4} z} v_{4}^{l} \boldsymbol{v}_{4} .
$$

In the vacuum limit $\varepsilon_{\mu \nu}=\delta_{\mu \nu}+\epsilon \alpha_{\mu \nu}$ and $k_{3}^{(1,2)}=+k_{z}+$ $\epsilon \delta k_{3}^{(1,2)}, k_{3}^{(3,4)}=-k_{z}+\epsilon \delta k_{3}^{(3,4)}$ we obtain

$$
\begin{array}{ll}
\boldsymbol{v}_{1} \rightarrow \boldsymbol{v}_{1}^{0}+c_{1} \boldsymbol{v}_{2}^{0}, & \boldsymbol{v}_{2} \rightarrow c_{2} \boldsymbol{v}_{1}^{0}+\boldsymbol{v}_{2}^{0}, \\
\boldsymbol{v}_{3} \rightarrow \boldsymbol{v}_{3}^{0}+c_{3} \boldsymbol{v}_{4}^{0}, & \boldsymbol{v}_{4} \rightarrow c_{4} \boldsymbol{v}_{3}^{0}+\boldsymbol{v}_{4}^{0},
\end{array}
$$

where

$$
\begin{aligned}
& c_{1}=-\frac{\omega^{2}\left(k_{z} \alpha_{21}-k_{1} \alpha_{23}\right)+k_{2}\left(k_{1} \zeta_{3}-k_{z} \zeta_{1}\right)}{\omega^{2}\left(k_{z} \alpha_{22}-k_{2} \alpha_{23}\right)+k_{2}\left(k_{2} \zeta_{3}-k_{z} \zeta_{2}\right)-2 k_{z}^{2} \delta k_{3}^{(1)}}, \\
& c_{2}=-\frac{\omega^{2}\left(k_{z} \alpha_{12}-k_{2} \alpha_{13}\right)+k_{1}\left(k_{2} \zeta_{3}-k_{z} \zeta_{2}\right)}{\omega^{2}\left(k_{z} \alpha_{11}-k_{1} \alpha_{13}\right)+k_{1}\left(k_{1} \zeta_{3}-k_{z} \zeta_{1}\right)-2 k_{z}^{2} \delta k_{3}^{(2)}}, \\
& c_{3}=\left.c_{1}\right|_{k_{z} \rightarrow-k_{z}, \delta k_{3}^{(1)} \rightarrow \delta k_{3}^{(3)}}, \\
& c_{4}=\left.c_{2}\right|_{k_{z} \rightarrow-k_{z}, \delta k_{3}^{(2)} \rightarrow \delta k_{3}^{(4)}},
\end{aligned}
$$

and $\zeta_{i}=k^{n} \alpha_{n i}$. We note that the eigenvectors (A9) have been chosen in a form such that in the vacuum limit these eigenvectors become a linear combination of vacuum vectors, in agreement with quantum mechanical perturbation theory in the degenerate case.

\section{Scattering problem}

Let us consider now a general scattering problem with matter described by dielectric permittivity $\varepsilon_{\mu \nu}$ to the left (index l) of the boundary $z=0$ and vacuum, $\varepsilon_{\mu \nu}=\delta_{\mu \nu}$, to the right (index $r$ ). The field has the structure to the left of the boundary (inside matter)

$$
\begin{aligned}
& \mathbf{E}_{l}=e^{i \kappa_{1} z} A_{i}^{l} \mathcal{E}_{1}^{l}+e^{i \kappa_{2} z} B_{i}^{l} \mathcal{E}_{2}^{l}+e^{i \kappa_{3} z} A_{o}^{l} \mathcal{E}_{3}^{l}+e^{i \kappa_{4} z} B_{o}^{l} \mathcal{E}_{4}^{l}, \\
& \mathbf{H}_{l}=e^{i \kappa_{1} z} A_{i}^{l} \mathcal{H}_{1}^{l}+e^{i \kappa_{2} z} B_{i}^{l} \mathcal{H}_{2}^{l}+e^{i \kappa_{3} z} A_{o}^{l} \mathcal{H}_{3}^{l}+e^{i \kappa_{4} z} B_{o}^{l} \mathcal{H}_{4}^{l},
\end{aligned}
$$

and to the right of the boundary (in vacuum)

$$
\begin{aligned}
\mathbf{E}_{r}= & e^{i k_{z} z} A_{o}^{r} \mathcal{E}_{1}^{r}+e^{i k_{z} z} B_{o}^{r} \mathcal{E}_{2}^{r} \\
& +e^{-i k_{z} z} A_{i}^{r} \mathcal{E}_{3}^{r}+e^{-i k_{z} z} B_{i}^{r} \mathcal{E}_{4}^{r}, \\
\mathbf{H}_{r}= & e^{i k_{z} z} A_{o}^{r} \mathcal{H}_{1}^{r}+e^{i k_{z} z} B_{o}^{r} \mathcal{H}_{2}^{r} \\
& +e^{-i k_{z} z} A_{i}^{r} \mathcal{H}_{3}^{r}+e^{-i k_{z} z} B_{i}^{r} \mathcal{H}_{4}^{r},
\end{aligned}
$$

where the subscript $i(o)$ denotes incoming (outgoing) waves on the boundary, and $\kappa_{n}, n=1,2,3,4$ are solutions of (A6). We have eight amplitudes, $A_{i, o}^{l, r}, B_{i, o}^{l, r}$, to be defined. They are related by the scattering matrix which is defined through boundary (matching) conditions.

The in and out states and $\mathbf{S}$ matrix read

$$
\mathbf{E}^{\text {out }}=\left(\begin{array}{c}
A_{o}^{l} \\
B_{o}^{l} \\
A_{o}^{r} \\
B_{o}^{r}
\end{array}\right), \quad \mathbf{E}^{\text {in }}=\left(\begin{array}{c}
A_{i}^{l} \\
B_{i}^{l} \\
A_{i}^{r} \\
B_{i}^{r}
\end{array}\right), \quad \mathbf{E}^{\text {out }}=\mathbf{S} \cdot \mathbf{E}^{\text {in }},
$$

where

$$
\mathbf{S}=\left(\begin{array}{cc}
\mathbf{r} & \mathbf{t}^{\prime} \\
\mathbf{t} & \mathbf{r}^{\prime}
\end{array}\right), \quad \mathbf{r}=\left(\begin{array}{ll}
r_{x x} & r_{x y} \\
r_{y x} & r_{y y}
\end{array}\right), \quad \mathbf{t}=\left(\begin{array}{cc}
t_{x x} & t_{x y} \\
t_{y x} & t_{y y}
\end{array}\right)
$$

To obtain the $\mathbf{S}$ matrix we use the boundary conditions

$$
\begin{aligned}
\left(\mathbf{E}^{l}-\mathbf{E}^{r}\right) \times\left.\boldsymbol{n}_{l \rightarrow r}\right|_{z=0} & =\mathbf{0}, \\
\left(\mathbf{H}^{l}-\mathbf{H}^{r}\right) \times\left.\boldsymbol{n}_{l \rightarrow r}\right|_{z=0} & =\left.4 \pi \boldsymbol{\sigma}_{s} \mathbf{E}^{r}\right|_{z=0},
\end{aligned}
$$

where $\sigma_{s}$ is the (possible) tensor conductivity on the boundary $z=0$.

We use the boundary conditions (A15). Because $\boldsymbol{n}=$ $(0,0,1)$ we may rewrite these equations in the following form:

$$
\boldsymbol{e}^{l}=\boldsymbol{e}^{r}, \quad \boldsymbol{h}^{l}=\boldsymbol{h}^{r}+\frac{4 \pi}{c}\left(\widetilde{\boldsymbol{\sigma}}_{s} \boldsymbol{e}^{r}\right),
$$

where $\boldsymbol{e}, \boldsymbol{h}$ are from Eq. (A4) and

$$
\tilde{\boldsymbol{\sigma}}_{s}=\left(\begin{array}{cc}
-\sigma_{21} & -\sigma_{22} \\
\sigma_{11} & \sigma_{12}
\end{array}\right) .
$$


Taking into account Eqs. (A8) and (A10), the boundary conditions are represented in the following form:

$A_{o}^{l} \boldsymbol{v}_{3}^{l}+B_{o}^{l} \boldsymbol{v}_{4}^{l}-A_{o}^{r} \hat{\boldsymbol{v}}_{1}^{r}-B_{o}^{r} \hat{\boldsymbol{v}}_{2}^{r}=-A_{i}^{l} \boldsymbol{v}_{1}^{l}-B_{i}^{l} \boldsymbol{v}_{2}^{l}+A_{i}^{r} \hat{\boldsymbol{v}}_{3}^{r}+B_{i}^{r} \hat{\boldsymbol{v}}_{4}^{r}$.

By solving these relations we obtain components of the $\mathbf{S}$ matrix:

$$
\begin{aligned}
& \mathbf{r}=-\frac{1}{\Delta}\left(\begin{array}{llllllll}
\mid \boldsymbol{v}_{1}^{l} & \boldsymbol{v}_{4}^{l} & \hat{\boldsymbol{v}}_{1}^{r} & \hat{\boldsymbol{v}}_{2}^{r} \mid & \mid \boldsymbol{v}_{2}^{l} & \boldsymbol{v}_{4}^{l} & \hat{\boldsymbol{v}}_{1}^{r} & \hat{\boldsymbol{v}}_{2}^{r} \mid \\
\mid \boldsymbol{v}_{3}^{l} & \boldsymbol{v}_{1}^{l} & \hat{\boldsymbol{v}}_{1}^{r} & \hat{\boldsymbol{v}}_{2}^{r} \mid & \mid \boldsymbol{v}_{3}^{l} & \boldsymbol{v}_{2}^{l} & \hat{\boldsymbol{v}}_{1}^{r} & \hat{\boldsymbol{v}}_{2}^{r} \mid
\end{array}\right), \\
& \mathbf{t}^{\prime}=+\frac{1}{\Delta}\left(\begin{array}{llllllll}
\mid \hat{\boldsymbol{v}}_{3}^{r} & \boldsymbol{v}_{4}^{l} & \hat{\boldsymbol{v}}_{1}^{r} & \hat{\boldsymbol{v}}_{2}^{r} \mid & \mid \hat{\boldsymbol{v}}_{4}^{r} & \boldsymbol{v}_{4}^{l} & \hat{\boldsymbol{v}}_{1}^{r} & \hat{\boldsymbol{v}}_{2}^{r} \mid \\
\mid \boldsymbol{v}_{3}^{l} & \hat{\boldsymbol{v}}_{3}^{r} & \hat{\boldsymbol{v}}_{1}^{r} & \hat{\boldsymbol{v}}_{2}^{r} \mid & \mid \boldsymbol{v}_{3}^{l} & \hat{\boldsymbol{v}}_{4}^{r} & \hat{\boldsymbol{v}}_{1}^{r} & \hat{\boldsymbol{v}}_{2}^{r} \mid
\end{array}\right), \\
& \mathbf{t}=+\frac{1}{\Delta}\left(\begin{array}{llllllll}
\mid \boldsymbol{v}_{3}^{l} & \boldsymbol{v}_{4}^{l} & \boldsymbol{v}_{1}^{l} & \hat{\boldsymbol{v}}_{2}^{r} \mid & \mid \boldsymbol{v}_{3}^{l} & \boldsymbol{v}_{4}^{l} & \boldsymbol{v}_{2}^{l} & \hat{\boldsymbol{v}}_{2}^{r} \mid \\
\mid \boldsymbol{v}_{3}^{l} & \boldsymbol{v}_{4}^{l} & \hat{\boldsymbol{v}}_{1}^{r} & \boldsymbol{v}_{1}^{l} \mid & \mid \boldsymbol{v}_{3}^{l} & \boldsymbol{v}_{4}^{l} & \hat{\boldsymbol{v}}_{1}^{r} & \boldsymbol{v}_{2}^{l} \mid
\end{array}\right), \\
& \mathbf{r}^{\prime}=-\frac{1}{\Delta}\left(\begin{array}{llllllll}
\mid \boldsymbol{v}_{3}^{l} & \boldsymbol{v}_{4}^{l} & \hat{\boldsymbol{v}}_{3}^{r} & \hat{\boldsymbol{v}}_{2}^{r} \mid & \mid \boldsymbol{v}_{3}^{l} & \boldsymbol{v}_{4}^{l} & \hat{\boldsymbol{v}}_{4}^{r} & \hat{\boldsymbol{v}}_{2}^{r} \mid \\
\mid \boldsymbol{v}_{3}^{l} & \boldsymbol{v}_{4}^{l} & \hat{\boldsymbol{v}}_{1}^{r} & \hat{\boldsymbol{v}}_{3}^{r} \mid & \mid \boldsymbol{v}_{3}^{l} & \boldsymbol{v}_{4}^{l} & \hat{\boldsymbol{v}}_{1}^{r} & \hat{\boldsymbol{v}}_{4}^{r} \mid
\end{array}\right),
\end{aligned}
$$

where

$$
\Delta=\left|\boldsymbol{v}_{3}^{l} \boldsymbol{v}_{4}^{l} \hat{\boldsymbol{v}}_{1}^{r} \hat{\boldsymbol{v}}_{2}^{r}\right|, \quad \hat{\boldsymbol{v}}_{n}^{r}=\boldsymbol{v}_{n}^{r}+\boldsymbol{v}_{n}^{\sigma}, \quad \boldsymbol{v}_{n}^{\sigma}=\left(\begin{array}{c}
\mathbf{0} \\
\frac{4 \pi}{c} \tilde{\boldsymbol{\sigma}}_{s} \boldsymbol{e}_{n}^{r}
\end{array}\right),
$$

and the vertical lines mean determinant.

\section{Rarefication procedure}

To rarefy matter we use the relation $\varepsilon_{\mu \nu}=\delta_{\mu \nu}+4 \pi N \alpha_{\mu \nu}$ and expand the expression for $\mathbf{r}^{\prime}$ up to first order in $N$. After long calculations we obtain

$$
\begin{aligned}
r_{i j}^{\prime}= & \frac{\pi N}{k_{z}^{2}}\left(\alpha_{33} k_{i} k_{j}-\omega^{2} \alpha_{i j}+k_{i} \alpha_{n j} k^{n}\right) \\
& +\frac{\pi N}{k_{z}^{3}}\left[k_{i} k_{j} \alpha_{n 3} k^{n}-\boldsymbol{k}^{2} \alpha_{i 3} k_{j}+k_{z}^{2}\left(k_{i} \alpha_{3 j}-\alpha_{i 3} k_{j}\right)\right] .
\end{aligned}
$$

Because

$$
\ln \operatorname{det}(\mathbf{I}-\epsilon \mathbf{A})=1-\epsilon \operatorname{tr} \mathbf{A}+O\left(\epsilon^{2}\right),
$$

from (2) we obtain

$$
\mathcal{E}_{\mathrm{C}}=-\iint \frac{d^{2} k}{2(2 \pi)^{3}} \int_{-\infty}^{\infty} d \xi e^{-2 a \kappa} \operatorname{tr}\left(\mathbf{r}^{\prime} \mathbf{r}\right)+O\left(N^{2}\right) .
$$

Straightforward calculation gives

$$
\begin{aligned}
\operatorname{tr}\left(\mathbf{r}^{\prime} \mathbf{r}\right)= & -\frac{\pi N}{\kappa^{2}} r^{i j}\left\{\xi^{2} \alpha_{j i}+\alpha_{n i} k^{n} k_{j}+\alpha_{33} k_{i} k_{j}\right\} \\
& -\operatorname{sgn}(\xi) \frac{i \pi N}{\kappa^{3}} r^{i j}\left\{\kappa^{2}\left[\alpha_{3 i} k_{j}-\alpha_{j 3} k_{i}\right]\right. \\
& \left.-k_{i}\left(\boldsymbol{k}^{2} \alpha_{j 3}-\alpha_{n 3} k^{n} k_{j}\right)\right\} .
\end{aligned}
$$

The last imaginary term gives no contribution to the energy and we arrive at the expression

$$
\begin{aligned}
\mathcal{E}_{\mathrm{CP}}= & \iint \frac{d^{2} k}{(2 \pi)^{2}} \int_{0}^{\infty} \frac{d \xi}{\kappa} e^{-2 \kappa a} r^{i j} \\
& \times\left[\xi^{2} \alpha_{j i}+\alpha_{n i} k^{n} k_{j}+\alpha_{33} k_{i} k_{j}\right] .
\end{aligned}
$$

[1] M. Bordag, G. L. Klimchitskaya, U. Mohideen, and V. M. Mostepanenko, Advances in the Casimir Effect (Oxford University Press, Oxford, 2009), p. 768.

[2] A. V. Parsegian, Van der Waals Forces. A Handbook for Biologists, Chemists, Engineers, and Physicists (Cambridge University Press, Cambridge, 2006), p. 380.

[3] P. W. Milonni, The Quantum Vacuum: An Introduction to Quantum Electrodynamics. (Academic, New York, 1994), p. 522.

[4] L. M. Woods, D. A. R. Dalvit, A. Tkatchenko, P. RodriguezLopez, A. W. Rodriguez, and R. Podgornik, Rev. Mod. Phys. 88, 045003 (2016).

[5] H. B. G. Casimir, Proc. K. Ned. Akad. Wet. 51, 793 (1948).

[6] R. Messina, P. A. Maia Neto, B. Guizal, and M. Antezza, Phys. Rev. A 92, 062504 (2015).

[7] R. Messina and M. Antezza, Phys. Rev. A 89052104 (2014).

[8] M. Antezza, L. P. Pitaevskii, and S. Stringari, Phys. Rev. Lett. 95, 113202 (2005).

[9] M. Antezza, L. P. Pitaevskii, S. Stringari, and V. B. Svetovoy, Phys. Rev. A 77, 022901 (2008).

[10] R. Messina and M. Antezza, Phys. Rev. A 84, 042102 (2011).

[11] A. Noto, R. Messina, B. Guizal, and M. Antezza, Phys. Rev. A 90, 022120 (2014).

[12] J. M. Obrecht, R. J. Wild, M. Antezza, L. P. Pitaevskii, S. Stringari, and E. A. Cornell, Phys. Rev. Lett. 98, 063201 (2007).

[13] M. Bordag, I. V. Fialkovsky, D. M. Gitman, and D. V. Vassilevich, Phys. Rev. B 80, 245406 (2009).

[14] C. Abbas, B. Guizal, and M. Antezza, Phys. Rev. Lett. 118, 126101 (2017)
[15] A. G. Grushin and A. Cortijo, Phys. Rev. Lett. 106, 020403 (2011); B.-S. Lu, Phys. Rev. B 97, 045427 (2018).

[16] S. Fuchs, F. Lindel, R. V. Krems, G. W. Hanson, M. Antezza, and S. Y. Buhmann, Phys. Rev. A 96, 062505 (2017).

[17] R. Zhao, J. Zhou, T. Koschny, E. N. Economou, and C. M. Soukoulis, Phys. Rev. Lett. 103, 103602 (2009).

[18] J. H. Wilson, A. A. Allocca, and V. Galitski, Phys. Rev. B 91, 235115 (2015); P. Rodriguez-Lopez, A. Popescu, I. Fialkovsky, N. Khusnutdinov, and L. M. Woods, Commun. Mater. 1, 14 (2020).

[19] H. B. G. Casimir and D. Polder, Phys. Rev. 73, 360 (1948).

[20] F. Intravaia, C. Henkel, and M. Antezza, in Casimir Physics, edited by D. Dalvit, P. Milonni, D. Roberts, and F. da Rosa, Lecture Notes in Physics (Springer, Berlin, 2011), Vol. 834.

[21] E. M. Lifshitz, Sov. Phys. JETP 2, 73 (1956).

[22] J. F. Babb, J. Phys.: Conf. Ser. 19, 1 (2005); K. V. Shajesh and M. Schaden, Phys. Rev. A 85, 012523 (2012); P. Thiyam, P. Parashar, K. V. Shajesh, O. I. Malyi, M. Boström, K. A. Milton, I. Brevik, and C. Persson, Phys. Rev. Lett. 120, 131601 (2018); S. Y. Buhmann, V. N. Marachevsky, and S. Scheel, Phys. Rev. A 98, 022510 (2018).

[23] P. Thiyam, P. Parashar, K. V. Shajesh, C. Persson, M. Schaden, I. Brevik, D. F. Parsons, K. A. Milton, O. I. Malyi, and M. Boström, Phys. Rev. A 92, 052704 (2015).

[24] V. N. Marachevsky and Y. M. Pis'mak, Phys. Rev. D 81, 065005 (2010).

[25] V. A. Parsegian and G. H. Weiss, J. Adhes. 3, 259 (1972); Y. S. Barash, Radiophys. Quantum Electron. 21, 1138 (1978). 
[26] S. A. H. Gangaraj, M. G. Silveirinha, G. W. Hanson, M. Antezza, and F. Monticone, Phys. Rev. B 98, 125146 (2018).

[27] L. P. Berestetskii, V. B. and Lifshitz, E. M. and Pitaevskii, Quantum Electrodynamics (Elsevier, Amsterdam, 1996), p. 667.

[28] A. M. Baldin and S. F. Semenko, Sov. Phys. JETP 12, 306 (1961); N. Manakov, V. Ovsiannikov, and L. Rapoport, Phys. Rep. 141, 320 (1986); J. H. Becher, S. Baier, K. Aikawa, M. Lepers, J.-F. Wyart, O. Dulieu, and F. Ferlaino, Phys. Rev. A 97, 012509 (2018).

[29] L. Zhizhimov and I. B. Khriplovich, Sov. Phys. JETP 55, 601 (1982).

[30] J. Mitroy, M. S. Safronova, and C. W. Clark, J. Phys. B: At. Mol. Opt. Phys. 43, 202001 (2010).

[31] L. Huang, M. Zhang, C. Li, and G. Shi, J. Phys. Chem. Lett. 6, 2806 (2015).

[32] D. Cohen-Tanugi and J. C. Grossman, Nano Lett. 12, 3602 (2012).

[33] M.-T. Jaekel and S. Reynaud, J. Phys. I 1, 1395 (2001).

[34] W. K. Tse and A. H. MacDonald, Phys. Rev. Lett. 109, 236806 (2012).
[35] I. Fialkovsky, N. Khusnutdinov, and D. Vassilevich, Phys. Rev. B 97, 165432 (2018).

[36] N. Khusnutdinov, R. Kashapov, and L. M. Woods, Phys. Rev. A 94, 012513 (2016); I. V. Fialkovsky, V. N. Marachevsky, and D. V. Vassilevich, Phys. Rev. B 84, 035446 (2011); M. Bordag, I. Fialkovskiy, and D. Vassilevich, ibid. 93, 075414 (2016).

[37] N. Khusnutdinov and N. Emelianova, Int. J. Mod. Phys. A 34, 1950008 (2019).

[38] T. Cysne, W. J. M. Kort-Kamp, D. Oliver, F. A. Pinheiro, F. S. S. Rosa, and C. Farina, Phys. Rev. A 90, 052511 (2014).

[39] F. Le Kien, P. Schneeweiss, and A. Rauschenbeutel, Eur. Phys. J. D 67, 92 (2013).

[40] N. Emelianova, I. V. Fialkovsky, and N. Khusnutdinov, Mod. Phys. Lett. A 35, 2040012 (2020).

[41] C. Henkel, G. L. Klimchitskaya, and V. M. Mostepanenko, Phys. Rev. A 97, 032504 (2018).

[42] A. Ali, R. Pothu, S. H. Siyal, S. Phulpoto, M. Sajjad, and K. H. Thebo, Mater. Sci. Energy Technol. 2, 83 (2019).

[43] B. M. Yoo, J. E. Shin, H. D. Lee, and H. B. Park, Curr. Opin. Chem. Eng. 16, 39 (2017).

[44] F. Zhou, M. Fathizadeh, and M. Yu, Annu. Rev. Chem. Biomol. Eng. 9, 17 (2018). 